\title{
Comparisons of Geomagnetic Transmission Measurements with Modified Tsyganenko 1989 Model Calculations for the October 1989 Solar Energetic Particle Events
}

\author{
P.R. Boberg* \\ Consultant, 27338 Brighton Lane, Valencia, CA 91354, USA. \\ D.F. Smart and M.A. Shea \\ 100 Tennyson Avenue, Nashua, NH 03062, USA. \\ A.J. Tylka \\ Code 672, NASA/Goddard Space Flight Center, Greenbelt, MD 20771, USA.
}

\begin{abstract}
We have determined eight-second averaged geomagnetic transmissions of 36-80 MeV protons for the large Solar Energetic Particle (SEP) events and geomagnetic activity level variations of October 1989 using measurements from the NOAA-10 and GOES-7 satellites. We have compared the geomagnetic transmission measurements with model calculations employing trajectory tracings through the combined International Geomagnetic Reference Field (IGRF) and Kp/Dst modified 1989 Tsyganenko model. We present threshold geomagnetic transmission geographic latitudes and magnetic latitudes, as well as (a) differences between the measured and calculated
\end{abstract}

\footnotetext{
*Corresponding author.: Tel. +1661 2639720.

Email addresses: pboberg@att.net (P.R. Boberg), sssrc@msn.com (D.F. Smart and M.A. Shea), allan.j.tylka@nasa.gov (A.J. Tylka)

Preprint submitted to Advances in Space Research

September 23, 2015
}

(C) 2015. This manuscript version is made available under the Elsevier user license http://www.elsevier.com/open-access/userlicense/1.0/ 
threshold geographic latitudes and magnetic latitudes and (b) differences between measured and calculated polar pass durations. We find that for less disturbed geomagnetic activity levels, the measured threshold geomagnetic transmission geographic and magnetic latitudes are typically about $1-1.5^{\circ}$ equatorward of the calculated geographic and magnetic latitudes, while for larger geomagnetic activity levels, the measured geographic and magnetic latitudes can be about $1.5^{\circ}$ poleward of the calculated geographic and magnetic latitudes. For the eight Kp bins, we also compare the mean measured magnetic latitudes as a function of mean Dst with the mean calculated magnetic latitudes, interpolated to the mean measured Dst values. These comparisons of mean magnetic latitudes illustrate the improvement in the accuracy of the model calculations resulting from employing the actual mean measured Dst values.

Keywords: geomagnetic cutoff rigidities, cutoff latitudes, magnetic fields, space radiation, space weather, solar particles

\section{Introduction}

Large Solar Energetic Particle events (SEP events) can produce ionic radiation extending into the tens to hundreds of Million electron Volts (MeVs) or greater (Aschwanden, 2012; Nitta et al., 2012; Shea \& Smart, 2012). These events are thought to be caused by coronal mass ejection (CME) driven shock acceleration of ambient solar wind material (Li et al., 2012; Reames, 2013) and suprathermal remnant solar flare material (Aschwanden, 2012; Gopalswamy et al., 2012; Kahler et al., 2012; Mewaldt et al., 2012; Reames, 2013; Tylka et al., 2001, 2005). The resultant SEPs can penetrate into the Earth's 
magnetosphere and (a) be detected by spacecraft detectors and (b) cause ionizing radiation effects to spacecraft instrumentation and materials (Tylka et al., 1997). The penetration of these SEPs into the magnetosphere has also been employed to measure heavy-ion SEP mean ionic charge states (Tylka et al., 1995; Leske et al., 1995; Mason et al., 1995; Oetliker et al., 1997; Mazur \& Mason, 2001; Labrador et al., 2003, 2005; Klecker et al., 2003).

These large SEP events can be accompanied by geomagnetic activity levels ranging from quiet to large geomagnetic disturbances (Boberg et al., 1995; Leske et al., 2001; Smart et al., 2006). The level of geomagnetic activity affects how deeply these SEPs penetrate into the magnetosphere. Previously, examinations of three-hour averaged proton geomagnetic transmissions were made and compared with model calculations (Boberg et al., 1995), including trajectory tracings through the combined International Geomagnetic Reference Field (IGRF) (Langel, 1991) and the 1989 Tsyganenko model (Tsyganenko, 1989) after the method of Flueckiger et al. (1991), including modifications to high levels of geomagnetic activity based on the hourly Dst geomagnetic activity indices. Since our nomenclature for geomagnetic transmission measurements discussed throughout this work can be in addition to standard geomagnetic cutoff nomenclature, we have included Table 1 to clarify our nomenclature.

More recently, geomagnetic cutoff measurements have been made for several SEP events, employing 8-15 MeV/nuc SAMPEX/MAST Helium data (Leske et al., 2001). We have compared plots of these measured geomagnetic cutoffs with model calculations (Smart et al., 2006) that included the IGRF and a Kp/Dst geomagnetic activity extension of the 1989 Tsyganenko model, 
using the three hour Kp geomagnetic activity indices to select the Kp/Dst based levels (also described in the Geomagnetic Transmission Modeling section later in this paper). We found a general level of agreement between the geomagnetic cutoff measurements and modeling of approximately 1 degree of invariant magnetic latitude for less geomagnetically disturbed intervals, and up to several degrees of invariant magnetic latitude for larger geomagnetically disturbed levels. We also found reasonable agreement between dose calculations and measurements obtained onboard the STS28 space shuttle flight (provided by NASA Johnson Space Center (JSC)) during the less disturbed geomagnetic activity levels of the August 1989 SEP event (Smart \& Shea, 2003; Smart et al., 2003, 2006).

Birch et al. (2005), using 35-70 MeV proton Northern hemisphere measurements from POES15 and POES16 for proton events during 2001, made comparisons between the measured cutoff magnetic latitudes and calculated cutoff magnetic latitudes employing the Tsyganenko (2002) magnetospheric model (Tsyganenko, 2002a,b). For quiet times to modestly disturbed intervals, the measurements and model calculations agreed to within $2 \sigma$ in cutoff magnetic latitude. This corresponds to the model calculations and measurements being comparable near Dst $=0 \mathrm{nT}$, with the model calculations' magnetic latitudes being $1^{\circ}-2^{\circ}$ poleward of the measurements near Dst=$100 \mathrm{nT}$, and model calculations' magnetic latitudes being around $5^{\circ}$ poleward of the measurements near Dst=-300 nT. Both of these POES satellites sample two local times during the Northern hemisphere passes (one local time for polar pass entry, and one local time for polar pass exit), providing four sets of local time cutoff measurements (local times of approximately 3, 8:30, 
12:30, and 18 hours). After comparing the measured cutoff magnetic latitudes with the Dst dependent Tsyganenko (2002) model calculations, Birch et al. (2005) also performed two variable linear regressions (Dst and magnetic latitude offset) for each of the four local times for the combination of the two geomagnetically active events, with correlation coefficients between 0.89 - 0.92. In addition, the standard errors of the four fits ranged from $\pm 0.9^{\circ}$ to $\pm 1.2^{\circ}$, while the magnetic latitude offsets varied by about $1.6^{\circ}$, and the slopes of the Dst dependence for all fits agreed within about 10\%. Specifically, the magnetic latitude offsets were in the range between $62.0^{\circ}$ and $63.6^{\circ}$, depending on local time, and the slopes of the Dst term varied between 0.027 and 0.029. Similar results were found employing the SYM-H (Kyoto WDC, 2015; Wanliss \& Showalter, 2006) geomagnetic activity index, instead of Dst. The range of these four local time, Dst fit results are useful for comparison with other local time average fits, as well as local time binned fits, described later in this Introduction, as well as for comparison with results we present in this paper.

Imaging riometer (IRIS) measurements for Halley, Antarctica $(L \approx 4.5)$ have been compared with calculations (Rodger et al., 2006) from universal time averaged geomagnetic cutoff calculations for earlier Kp/Dst based modeling (Smart \& Shea, 2003). These comparisons resulted in relatively good agreement between IRIS measurements and model calculations for quieter geomagnetic activity level intervals and cutoffs similar to the $\mathrm{Kp}=6$ calculations for larger geomagnetic disturbances. For less disturbed conditions, the measurements and modeling agree within what appears to us to be the estimated $0.2^{\circ}-0.3^{\circ}$ uncertainties taken from the more geomagnetically active 
times of the riometer analyses ${ }^{1}$, while for the largest geomagnetic activity levels, it appears that the magnetic latitudes of the model calculations are slightly more than $2^{\circ}$ equatorward of the measurements. Further imaging riometer measurements (Cliverd et al., 2007) from five SEP events indicate that the measurements and model calculations (Smart \& Shea, 2003) agree well and generally confirm their earlier results discussed above (Rodger et al., 2006), provided the model calculations for $\mathrm{Kp}=5$ are employed for larger geomagnetic disturbances, at least at rigidities of $>5$ to $>10 \mathrm{MeV}$ protons for those locations and conditions.

Neal et al. (2013) have performed empirical fits to geomagnetic cutoff measurements obtained using proton measurements from five POES satellites and the MetOp 02 satellite for fifteen SEP events during 2003 - 2012. Three proton energy intervals were considered, $16-35 \mathrm{MeV}, 35-70 \mathrm{MeV}$, and $70-140 \mathrm{MeV}$. Two types of empirical fits were considered for each energy interval. The first type of fit was quadratic in shifted Kp, where Kp from around three hours before the cutoff observations were employed in the empirical fits. The second type of empirical fit was linear in Dst. These energies and geomagnetic cutoffs were transformed to $100 \mathrm{~km}$, suitable for additional atmospheric calculations in their work, employing an altitude correction similar to the one we discuss in the geomagnetic transmission modeling section later in the paper. In general, the standard deviations of the fits ranged

\footnotetext{
${ }^{1}$ It also appears to us that the riometer modeling sensitivity to geomagnetic cutoff variations is lessened for $\mathrm{Kp} \leq 3$ values in these analyses, which would lead to larger uncertainties. The subsequent analyses (Cliverd et al., 2007) perhaps have more sensitivity to these lower levels of geomagnetic activity.
} 
between $1.1^{\circ}$ and $1.7^{\circ}$, while the magnitudes of the correlation coefficients were between 0.62 and 0.80 (the shifted $\mathrm{Kp}$ fits have negative correlation coefficients, since Kp increases as the geomagnetic cutoff latitude decreases). The linear Dst fit to the propagated $35-70 \mathrm{MeV}$ energy interval $(76.7 \mathrm{MeV}$ mean energy after propagation) has a magnetic latitude offset of $61.30^{\circ}$, and the slope of the Dst term is 0.02993. Neal et al. (2013) also confirmed that the geomagnetic cutoffs based on the modeling of (Smart \& Shea, 2003) are $1^{\circ}-2^{\circ}$ poleward of the polar satellite observations for most Kp geomagnetic activity levels, and that the model cutoffs from Smart \& Shea (2003) become equatorward shifted of the measured cutoffs for $\mathrm{Kp} \geq 8$.

For the 26-31 October 2003 SEP events, 19-27 MeV, 30-second averaged SAMPEX proton geomagnetic cutoff measurements have been compared (Kress et al., 2010) with interpolated lower-limit geomagnetic cutoff values $\left(\mathrm{R}_{L}\right)$ determined using the 2005 Tsyganenko model (Tsyganenko \& Sitnov, 2005). For periods including large geomagnetic disturbances, the measured cutoffs and the $\left(\mathrm{R}_{L}\right)$ calculations ${ }^{2}$ have a root-mean-square deviation of $1.8^{\circ}$ of magnetic latitude. In addition, the mean model, interpolated $\left(\mathrm{R}_{L}\right)$ calculations are $0.6^{\circ}$ of magnetic latitude poleward of the measured cutoffs. Recent comparisons between such model calculations and GOES particle fluxes for two proton energy intervals $(4.2 \mathrm{MeV}-8.7 \mathrm{MeV}$ and $8.7-14.5 \mathrm{MeV})$ from the December 2006 SEP event have shown East-West variations in which the model and measured fluxes qualitatively agree in shapes (Kress et al., 2013). They obtained improved agreement between measured and modeled fluxes if

\footnotetext{
${ }^{2} \mathrm{R}_{L}$ is the lowest allowed penumbral trajectory at a given location.
} 
the proton energies of the eastward cutoffs were reduced by $64 \%$ and $40 \%$ for the two energy channels, and if the proton energies of the westward cutoffs were reduced by $48 \%$ and $20 \%$ for the same channels.

Other low energy measurements from the polar orbiting POES 15-19 and MetOp 02 satellites during the January 2012 SEP event also show cutoff variations with Dst variations and day-night asymmetries (Nesse TyssoY et al., 2013). Most recently, Nesse TyssoY \& Stadsnes (2015) have performed Dst and interplanetary magnetic field based parameterizations of dayside and nightside geomagnetic cutoff latitude measurements from the available POES proton data for five energy intervals $(<2 \mathrm{MeV}, 2-6 \mathrm{MeV}, 6-10$ $\mathrm{MeV}, 10-20 \mathrm{MeV}$, and > $20 \mathrm{MeV}$ ) from six SEP events during 2003 - 2012. For the dayside intervals, the five parameterizations (one per energy interval) each employ three variables (Dst, Northward interplanetary magnetic field $\left(\mathrm{B}_{Z, N}\right)$, and magnetic latitude offset) to fit the cutoff measurements, and have correlation coefficents range from 0.65 - 0.75. For the nightside observations, two parameterizations (2 -6 MeV and $10-20 \mathrm{MeV}$ ) based on Dst, the third root of the interplanetary magnetic field pressure $(\rho)$, and magnetic latitude offset produce correlation coefficients of 0.70 and 0.72 , accounting for the fact that the magnetospheric tail field variations contribute to the cutoff changes for these low energy, nightside observations. The dayside slopes of the Dst component of these fits range from 0.090 for $<2 \mathrm{MeV}$ to 0.055 for $>20$ $\mathrm{MeV}$, significantly larger than the 0.027 - 0.030 Dst slopes of the $35-70$ MeV results from Birch et al. (2005) and Neal et al. (2013). The nightside slopes of the Dst component from Nesse TyssoY \& Stadsnes (2015) are more comparable to the Birch et al. (2005) and Neal et al. (2013) results, being 
0.040 and 0.035 for $2-6 \mathrm{MeV}$ and $10-20 \mathrm{MeV}$, respectively.

\section{Geomagnetic Transmission Measurements}

In order to measure the geomagnetic transmission during the large SEP events for 19.625 - 27.0 October 1989, we have employed proton data from NOAA-10 (815-837 km, dawn-dusk polar orbit) [NGDC (1995), Evans et al., private communication, 1995] and GOES-7 (geosynchronous orbit, effectively above the geomagnetic cutoffs at the proton energies considered) [NGDC (1994), R. Zwickl, private communication, 1994]. From NOAA-10, we have calculated eight-second averaged 36-80 MeV integral proton fluxes from the $>36 \mathrm{MeV}$ and $>80 \mathrm{MeV}$ integral channel data. For GOES-7, we determined five-minute averages of the $36-80 \mathrm{MeV}$ integral proton fluxes by calculating cubic splines of the $>30 \mathrm{MeV},>50 \mathrm{MeV},>60 \mathrm{MeV}$, and $>100$ $\mathrm{MeV}$ integral channel data. We then determined effective eight-second geomagnetic transmission averages by calculating the ratios of the $36-80 \mathrm{MeV}$ proton NOAA-10 to GOES-7 fluxes, giving high time resolution geomagnetic transmission measurements important for comparisons with modeling and radiation effects determinations. We estimate that the maximum systematic error in the measured geomagnetic transmissions from using five-minute averaged GOES-7 fluxes in lieu of eight-second averages is 15\%, including any short-lived SEP event rise phases, and that the typical systematic error is $<5 \%$. An example polar pass of the measured geomagnetic transmissions is shown in Figure 1. We have calculated the centroid orbital locations for each eight-second averaged geomagnetic transmission measurement by using the NOAA-10 orbital path (US Space Command, 1993). We present 
the absolute values of the interpolated geographic latitudes corresponding to threshold geomagnetic transmission measurements equal to $10 \%$ for both Northern and Southern hemispheres versus time in Figures 2.a through 9.a, presenting one day per figure. Throughout the rest of this paper, including figures and captions, we shall refer to the absolute values of the interpolated, threshold geomagnetic transmission geographic latitudes as simply geographic latitudes (this also applies to the model geomagnetic transmission calculations presented in the next section, Geomagnetic Transmission Modeling). Note, however, that absolute value signs for geographic latitudes are included in relevant labels of Figures 2.a-9.a (and 2.c-9.c, described later), as well as Figures 11 and 12. In Figures 2.a through 9.a, the effects of orbital dynamic variations on the geographic latitudes are clear, showing variations of $>25^{\circ}$, independent of geomagnetic activity disturbance levels (as shown in Figures 2.d through 9.d). We reiterate that there is a significant amount of nomenclature employed throughout this work, and we provide Table 1 as an aid.

In determining the geomagnetic transmission measurements, we have eliminated polar pass intervals with NOAA-10 or GOES-7 detector dead times, bad data, calibration data, and telemetry gaps on either entries or exits to the polar passes. We have also eliminated NOAA-10 South Atlantic Anomaly (SAA) periods from the geomagnetic transmission measurements. To eliminate small level statistical or penumbral oscillations near the $10 \%$ threshold geomagnetic transmission levels, we require that the identified polar passes have durations greater than two minutes.

We have calculated the McIlwain L parameters (McIlwain, 1961) for the 
1995.0 IGRF Epoch (Sabaka et al., 1997) at the centroid of each eight-second satellite location. We then calculated the invariant magnetic latitude (hereafter simply magnetic latitude) corresponding to each eight-second satellite location using:

$$
\cos ^{4} \Lambda=1 / L^{2}
$$

Finally, we interpolated the centroid eight-second magnetic latitudes to the magnetic latitudes corresponding to the $10 \%$ threshold geomagnetic transmission level. We refer to this as the measured magnetic latitude, $\Lambda$. As with the geographic latitudes, we shall refer to the interpolated, threshold geomagnetic transmission magnetic latitudes as simply magnetic latitudes throughout the rest of the paper. We present the magnetic latitudes in Figures 2.b through 9.b (unlike geographic latitudes shown in Figures 2.a through 9.a, magnetic latitudes are always positive, and thus do not need absolute value signs).

\section{Geomagnetic Transmission Modeling}

To begin the model calculations, we performed $5^{\circ}$ geographic latitude ${ }^{3}$ by $5^{\circ}$ geographic longitude reverse-direction, vertically incident trajectory tracings at $450 \mathrm{~km}$ altitude. The reverse-direction, vertically incident trajectory tracings employ a model magnetosphere consisting of the IGRF for epoch 1995.0 (Sabaka et al., 1997) and the 1989 Tsyganenko model (Tsyganenko, 1989) with the Dst-based modification for high geomagnetic activity

\footnotetext{
${ }^{3}$ Here, we refer to the actual geographic latitude of the model calculations. We return to the meaning given in Table 1 and elsewhere in this paper after the discussion of converting to calculated geomagnetic transmissions later in this section.
} 
levels (Flueckiger et al., 1991; Boberg et al., 1995; Smart et al., 2006). The reverse-direction trajectory tracings use the Bulirsch-Stoer numerical integration technique (Stoer and Bulirsch, 1980; Press et al., 1989).

At each $5^{\circ}$ geographic latitude by $5^{\circ}$ geographic longitude location, we have performed trajectory tracings, stepping down in $0.1 \mathrm{GV}$ rigidity increments high above the penumbra and in $0.01 \mathrm{GV}$ rigidity increments above and through the penumbra using techniques described in Smart et al. (2006). At each rigidity and location, the reverse-direction trajectory is determined allowed if it reached the model magnetospheric boundary or was considered to be a failed orbit if it had not reached any solution by 200,000 steps. The reverse-direction trajectory was determined forbidden if it intersected a model atmosphere set at $20 \mathrm{~km}$ above the Earth's surface.

At each rigidity step for a given location, we have defined a penumbral transparency as the ratio of allowed penumbral trajectories to total (allowed + forbidden) penumbral trajectories. To calculate an effective geomagnetic cutoff at each location, we have utilized the rigidity at which the penumbral transparency has fallen to 0.6. Since the NOAA-10 measurements are zenith pointing, we have employed the calculated vertical geomagnetic cutoffs in comparisons with the measurements.

The trajectory tracings employed in this work were performed for ten geomagnetic activity levels, six levels of $\mathrm{Kp}=0$ through $\mathrm{Kp}=5$ and four levels of $\mathrm{Kp}>5$ plus Dst $=-100 \mathrm{nT},-200 \mathrm{nT},-300 \mathrm{nT}$, and $-400 \mathrm{nT}$ (Smart et al., $2006)^{4}$. These modifications for the extreme values of Dst were motivated by

\footnotetext{
${ }^{4}$ We have also performed trajectory tracings for $\mathrm{Kp}>5$ plus Dst $=-500 \mathrm{nT}$, but it is unnecessary to employ those tracings in this work.
} 
estimates of the extraordinary large Dst values derived for the Carrington September 1859 magnetic activity (Tsurutani et al., 2003), e.g. that we should perform these calculations for geomagnetic activity levels beyond the October 1989 measurements, although these calculations still don't extend to the peak Dst estimates of the Carrington event. For our model calculations, we have assigned $\mathrm{Kp}=6$ through $\mathrm{Kp}=9$ values to the geomagnetic activity levels of $\mathrm{Kp}>5$ plus Dst $=-100 \mathrm{nT}$ through $\mathrm{Kp}>5$ plus Dst $=-400$ $\mathrm{nT}$. For each geomagnetic activity level, initial geomagnetic cutoff grids were calculated at every hour Universal Time (UT). In order to reduce the scatter in the hourly UT geomagnetic cutoff grids' penumbral variations, we then performed three-hour averagings of the hourly UT geomagnetic cutoff grids, e.g. averaging the $2 \mathrm{UT}, 3 \mathrm{UT}$, and $4 \mathrm{UT}$ hourly geomagnetic cutoff grids to produce the three-hour averaged, 3 UT geomagnetic cutoff grid. This produced eighty three-hour averaged, Kp/Dst geomagnetic activity binned cutoff grids employed here.

In order to determine the geomagnetic cutoffs corresponding to the centroid of the eight-second averaged NOAA-10 geomagnetic transmission measurements, we developed an interpolation/extrapolation procedure from the $5^{\circ}$ geographic latitude by $5^{\circ}$ geographic longitude, $450 \mathrm{~km}$ cutoff grids to arbitrary orbital locations. We begin by employing the relationship between vertical geomagnetic cutoff rigidities $\left(R_{C}\right)$ and McIlwain L parameter (McIlwain, 1961),

$$
R_{C}=V_{(k)} / L^{2} .
$$

In this relation, $V_{(k)}$ is the equivalent Stormer constant for the particular vertical geomagnetic cutoff rigidity. 
We have calculated geomagnetic cutoff $\left(R_{C}\right)$ values for computational and conceptual convenience using the conceptual $V_{(k)} / L^{2}$ interpolation where $V_{(k)}$ corresponds to the Stormer constant (Smart \& Shea, 1967).

For each satellite location, we first loaded the data parameters (McIlwain $\mathrm{L}$ values and cutoff rigidity values) for the four neighboring $450 \mathrm{~km}$ vertical geomagnetic cutoffs from the grids for the appropriate geomagnetic activity level and model time bins, and determined the $V_{(k)}$ values to form a grid around this satellite location. In this $450 \mathrm{~km}$ altitude plane, we then calculated the McIlwain L parameter for the satellite latitude for the left and right side of the grid and then calculated the $V_{(k)}$ parameters for these two positions. We then computed the McIlwain L parameter for the satellite position within this $450 \mathrm{~km}$ grid plane. Then, at the spacecraft latitude we interpolated the $V_{(k)}$ parameters to the spacecraft's geographic longitude within this grid plane by performing a weighted average of the variation in the McIlwain $\mathrm{L}$ parameter between the left and right side of the grid.

As a simple example, at a midway weighted interpolation point, the relationships of the interpolated values would be:

$$
\begin{aligned}
R_{C, \text { interp }} & =V_{(k, \text { interp })} / L_{\text {interp }}^{2} \\
& =1 / 2 *\left(V_{(1)} / L_{1}^{2}+V_{(2)} / L_{2}^{2}\right) \\
& =1 / 2 *\left(R_{C, 1}+R_{C, 2}\right) .
\end{aligned}
$$

We then used this $V_{(k)}$ value at $450 \mathrm{~km}$ and the McIlwain L parameter at the spacecraft's geographic latitude, geographic longitude, and altitude to extrapolate in altitude and thus calcuate the vertical $R_{C}$ at the spacecraft's location. 
After obtaining the geomagnetic cutoff at the satellite's location, we converted to an eight-second averaged 36-80 MeV geomagnetic transmission function by setting the calculated geomagnetic transmission to zero if the geomagnetic cutoff was above $80 \mathrm{MeV}$, setting the calculated geomagnetic transmission to unity if the geomagnetic cutoff was below $36 \mathrm{MeV}$, and setting the geomagnetic transmission to:

$$
\begin{aligned}
G T= & \left(F\left(E\left(R_{C}\right)\right)-F(80 \mathrm{MeV})\right) \\
& /(F(36 \mathrm{MeV})-F(80 \mathrm{MeV}))
\end{aligned}
$$

where $\mathrm{E}\left(R_{C}\right)$ is the proton energy corresponding to the calculated geomagnetic cutoff, $\mathrm{F}$ is the fit to the GOES-7 integral proton fluxes at that energy, and GT is the resultant calculated geomagnetic transmission. Figure 1 shows a sample polar pass comparison of these model calculations with the measured geomagnetic transmissions.

For direct comparisons with the measurements, we then have interpolated to the spacecraft positions corresponding to the threshold $10 \%$ level of the calculated geomagnetic transmissions for each polar pass entry and exit. We have eliminated any additional data gaps at the calculated threshold geomagnetic transmissions, as well as any threshold intervals eliminated from the measured threshold geomagnetic transmissions described previously. In Figures 2.a through 9.a, we show the resultant calculated geographic latitudes. Employing equation 1, we show the calculated magnetic latitudes in Figures 2.b through 9.b. 


\section{Discussion}

In Figures 2.a through 9.a, we show comparisons between the measured and calculated geographic latitudes for the Northern and Southern hemispheres as functions of time. The measured and calculated geographic latitudes track each other fairly well along the orbit but show some increasing deviation as the Kp geomagnetic activity levels increase. In Figures 2.b through 9.b, we show comparisons between the measured and calculated magnetic latitudes for the Northern and Southern hemispheres. Comparing these panels with Figures 2.a through 9.a, we find that the majority of the orbital dynamics' variations of the geographic latitudes corresponds to magnetic latitude deviations versus geographic latitudes across the globe. In other words, much of the geographic latitude variations are removed in the mapping to magnetic latitudes.

In order to present the entire duration of the SEP events succinctly, we show correlation plots comparing the measured and calculated magnetic latitudes for the Northern and Southern hemispheres in Figures 10 upper and lower, respectively. The differing Kp geomagnetic activity levels are shown by different symbols/colors. These figures show that that in addition to orbital dynamic variations seen in the geographic latitudes (Figures 2.a through 9.a), the magnetic latitudes vary with geomagnetic activity levels. These correlation plots and the comparisons shown in Figures 2.b through 9.b show that the cutoffs vary to a range of $\sim 10^{\circ}$ throughout the range of geomagnetic activity values from quiet to very disturbed.

We have computed the differences between measured and calculated geographic latitudes and between measured and calculated magnetic latitudes. 
Differences as functions of time are shown in Figures 2.c through 9.c. Positive (negative) differences correspond to the measured geographic latitudes or magnetic latitudes being poleward (equatorward) of the calculated geographic latitudes or magnetic latitudes. The differences of geographic latitudes tend to agree fairly well with the differences between magnetic latitudes. There are times when differences of geographic latitudes are substantially larger than the differences of magnetic latitudes, i.e. for some geomagnetically disturbed times (as seen in Figures 2.d through 9.d), when the measured geographic latitudes or magnetic latitudes are poleward of the calculations.

To quantify the differences between geographic latitudes and magnetic latitudes for the entire SEP events interval, we show histograms of these differences between measured and calculated geographic latitudes and between measured and calculated magnetic latitudes in Figure 11. The majority of these differences are between $\pm 2^{\circ}$ and the difference histograms peak near $-1^{\circ}$ to $-1.5^{\circ}$. These results show that the geographic latitude and magnetic latitude measurements agree reasonably well with the calculations and that the measured geographic latitudes and magnetic latitudes tend to be shifted somewhat equatorward of the calculated geographic latitudes and magnetic latitudes.

In Figure 12, we show Kp geomagnetic activity binned histograms of the differences between measured and calculated geographic latitudes, as well as between measured and calculated magnetic latitudes. These results show that for quieter geomagnetic activity levels, there are appreciable intervals in which the measured geographic latitudes and magnetic latitudes are shifted 
equatorward of the calculated ones. However, for larger geomagnetic activity intervals such as $\mathrm{Kp}=7$ and $\mathrm{Kp}=8$, we observe that the measured geographic latitudes and magnetic latitudes tend to be shifted poleward of the calculations, typically by $2^{\circ}$ poleward or less, but with a poleward shift of the measured geographic latitude compared to the calculated geographic latitude of up to $7.5^{\circ}$ in one extreme case.

We have calculated the differences between the measured and calculated times corresponding to the durations between each pair of polar pass threshold geomagnetic transmission occurrences. Such time differences are important in determining the accuracy of radiation exposure calculations. Positive time differences correspond to longer measured polar pass intervals than calculated ones, while negative time differences correspond to shorter measured polar pass intervals than the calculations. Histograms of the time differences for the entire SEP events interval and for Kp geomagnetic activity binned periods are shown in Figures 13 and 14, respectively. The measured durations of the polar passes shown in Figure 13 peak around 30 seconds longer than the calculated durations, and the vast majority of time differences for the polar passes are within \pm 2 minutes. Analogous to the geographic and magnetic latitude differences shown in Figure 12, Figure 14 shows that as Kp geomagnetic activity increases, there is a tendency towards polar passes with negative time differences, i.e. polar passes in which the calculated durations are longer than the measured durations, typically by less than 2 minutes.

We present the means of the overall and Kp binned geographic latitudes, magnetic latitudes, geographic latitude differences, magnetic latitude differences, standard deviations of the distributions for the magnetic latitude 
differences, Northern and Southern hemisphere magnetic latitude differences, and Dst values with standard deviations in Table 2. We also give the means of the overall and Kp binned polar pass time durations and corresponding differences with standard deviations in Table 3. The average of the magnetic latitude results over geomagnetic activity levels and both hemispheres shows that the mean difference between the measurements and model results is less than $0.5^{\circ}$, with a standard deviation of $1.5^{\circ}$. The geomagnetic activity dependent differences between magnetic latitude measurements and model calculations are within $\pm 1^{\circ}$ to $\pm 1.5^{\circ}$, with up to $-1.7^{\circ}$ differences for a couple of Northern hemisphere Kp bins, and \pm 1.1 minute for the polar pass duration time differences. The geomagnetic activity dependent variations (e.g. changes between positive and negative values) of $2.8^{\circ}$ magnetic latitude differences and 2 minutes time differences are also evident in Tables 2 and 3. In Table 3, it is also evident that the polar pass durations increase by 3 or more minutes between geomagnetically quiet and disturbed intervals. Examining only the geomagnetically less disturbed intervals, the measured polar pass durations illustrate that different orbital segments for these $\mathrm{Kp}$ bins cause some modest variation in the polar pass durations.

In this work, we have focused on the accuracy of the Kp/Dst-binned IGRF + modified Tsyganenko 1989 model calculations. Since three hour Kp and hourly Dst can vary independently, it is useful to examine the separate variations of these two geomagnetic activity parameters. One method for accomplishing this is to plot the variations in the differences of the magnetic latitudes for each Kp bin versus the Dst activity level corresponding to the measurements. We also can examine the results separately for the Northern 
and Southern hemisphere passes. Figure 15 presents these results. If the geomagnetic transmissions were described solely by $\mathrm{Kp}$, then the plotted differences in the magnetic latitudes for each $\mathrm{Kp}$ bin would be (a) clustered at specific values for each Kp-bin, and (b) independent of Dst. From Figure 15, in agreement with results presented earlier on the distributions of the differences in the magnetic latitudes, the data are not clustered at specific values for each Kp-bin, indicating that the results are not perfectly ordered by Kp. In addition, we note that for specific $\mathrm{Kp}$ and Dst values (e.g. for $\mathrm{Kp}=5$ and Dst=-100 nT) within a panel of Figure 15, the results still show a reasonable variation. Thus, although statistics do not allow us to quantify this effect in general, the results suggest an intrinsic variation, even for specific pairs of Kp and Dst values.

As far as the question on whether the Kp-binned results might be independent of Dst, Figure 15 shows that in general, for the Kp-bins, there are generally systematic variations in the magnetic latitude differences with Dst, ranging from variations on the order of one degree for the $\mathrm{Kp}=3$ and $\mathrm{Kp}=5$ bins to Dst variations of a couple degrees or more for some bins, such as $\mathrm{Kp}=4, \mathrm{Kp}=6$, and $\mathrm{Kp}=7$. It is not surprising that there are systematic variations with Dst levels, although it is unclear why some of the Kp-bins show different magnitude of variations when examining comparable Dst intervals. Perhaps a larger sample size of events and magnetic disturbances would clarify this issue. Figure 15 also illustrates that a residual systematic difference exists between the Northern and Southern hemisphere magnetic latitude differences, consistent with the mean values presented in Table 2. Furthermore, Figure 15 suggests that for specific Kp and Dst pairs, the total 
spread in the Northern or Southern hemisphere magnetic latitude differences is typically perhaps two degrees or less, although there are Kp and Dst pairs showing larger variations.

One suggested analysis would be to determine what Dst values in hypothetical model calculations would the mean magnetic latitude differences in Figure 15 be equal to zero. While the model calculations are not spaced sufficiently finely in Dst to make an accurate, direct determination, it is possible to employ a spline interpolation between the Dst values of the adjacent Kp/Dst model calculations to obtain approximate answers. Re-examining Table 2 , we note that the measured mean magnetic latitude for $\mathrm{Kp}=2$ roughly corresponds to the calculated mean magnetic latitude for $\mathrm{Kp}=4$. Although we haven't discussed this previously, it also turns out that for these SEP events, the measured Dst values for the Kp-bins given in Table 2 are typically larger than the corresponding Dst values employed in the model calculations (the two exceptions to this are the geomagnetic disturbed $\mathrm{Kp}=7$ and $\mathrm{Kp}=8$ bins). So, in the example comparison of the $\mathrm{Kp}=2$ measurements to $\mathrm{Kp}=4$ calculations, it turns out that a model Dst $=-40.5 \mathrm{nT}$ would approximately correspond to the $\mathrm{Kp}=2$ mean magnetic latitude measurement, which for the events analyzed here, corresponds to a mean measured Dst=-53 nT. By employing a spline interpolation (more detail on the complementary version of this technique is given in the next paragraph), the model calculation's Dst values needed to obtain approximate agreement with the measured mean magnetic latitudes are typically 5-20 $\mathrm{nT}$ smaller in magnitude (the absolute value of Dst) than the measured mean Dst values for $\mathrm{Kp} \leq 6$ values. For $\mathrm{Kp}=7$, the model and measured mean Dst values are essentially in agreement. 
Finally, the largest exception is for the $\mathrm{Kp}=8 \mathrm{bin}$, where the corresponding magnitude of the model calculation, mean Dst value is approximately $40 \mathrm{nT}$ greater than the mean measurement. It is worth noting that the systematic variations of the differences in magnetic latitudes as a function of Dst for the $\mathrm{Kp}=8$ bin shown in Figure 15 are not as pronounced as most other Kp bins. We will examine the Dst behavior of the $\mathrm{Kp}=8$ and other bins more closely when we present Figures 16 and 17 later in this work.

A complementary analysis to the above measurement and model Dst comparisons at the same magnetic latitudes is to compare the measured mean magnetic latitudes at the measured Dst values with the model mean magnetic latitudes, interpolated to the mean Dst values of the data. In this interpolation, the model Dst values for the eight Kp levels observed here correspond to the independent variables, and the model mean magnetic latitudes at these eight Kp levels correspond to the dependent variables. The resulting interpolations at the eight mean measured Dst values gives the eight model mean magnetic latitudes at the measured Dst values, and Figure 16 includes these results (labeled as Model, interpolated). In addition, Figure 16 includes the mean model magnetic latitudes at the model Dst values (labeled as Model), as well as the mean magnetic latitude data at the mean measured Dst values (labeled as Data). In an analogous manner, we also show the mean measurements for the Northern and Southern hemispheres separately, and finally the model mean magnetic latitudes at the model Dst values for the two hemispheres separately.

Returning to the conceptual point that the primary purpose of Figure 16 is to compare mean measured and model magnetic latitudes at the mean Dst 
values of the data, we see that the differences in mean magnetic latitudes range from $<0.1^{\circ}$ to about $0.7^{\circ}$. For $\mathrm{Kp} \leq 6$, the mean model magnetic latitudes are lower (equatorward) than the mean measured magnetic latitudes, with excellent agreement $\left(<0.2^{\circ}\right)$ for the $\mathrm{Kp}=1$ and $\mathrm{Kp}=3$ cases. In addition, there is excellent agreement for $\mathrm{Kp}=7$ as well, with the mean model magnetic latitude being $<0.1^{\circ}$ poleward of the mean measured magnetic latitude. For $\mathrm{Kp}=8$, the mean model magnetic latitude is about $0.7^{\circ}$ poleward of the mean measured magnetic latitude.

Turning to the individual hemispheres shown in Figure 16, it is clear that for the Northern hemisphere, the mean model and measured magnetic latitudes are in excellent agreement for $\mathrm{Kp} \leq 6$, with deviations ranging from $0^{\circ}$ to $0.3^{\circ}$, and which are typically $\leq 0.1^{\circ}$. For $\mathrm{Kp}=7$, the mean model magnetic latitude point is about $0.5^{\circ}$ poleward of the measurement mean. For $\mathrm{Kp}=8$, the mean model results are about $1^{\circ}$ poleward of the mean measured results.

In contrast, for $\mathrm{Kp} \leq 7$ in the Southern hemisphere, the mean model magnetic latitudes range from about $0.2^{\circ}$ to $1^{\circ}$ equatorward of the mean measured magnetic latitudes. For $\mathrm{Kp}=8$, the mean model results are $<0.4^{\circ}$ poleward of the mean measurements. It should be noted that Figure 16 also shows that for $\mathrm{Kp} \leq 6$, the mean magnetic latitude Southern hemisphere measurements modestly differ from the otherwise excellent clustering of the Northern hemisphere mean measurements, calculations, and Southern hemisphere calculations.

Although Figure 16 illustrates that neither the measured mean magnetic latitudes nor model mean magnetic latitudes have complete linear dependence on Dst over all geomagnetic activity levels, both types of mean mag- 
netic latitudes indicate rough linear dependence with Dst over the geomagnetic activity levels corresponding to $\mathrm{Kp}=1$ to $\mathrm{Kp}=7$. Therefore, we present the parameters of these linear Dst fits for the measured and model calculation magnetic latitudes for the two hemispheres in Table 4 (since these fits are to mean values, the correlation coefficents are $>0.98$ in all four cases). As suggested by the results in Figure 16, the measured and model fits agree with each other reasonably well for both hemispheres, with the Northern hemisphere showing better agreement than the Southern hemisphere. In all cases, the slopes of the Dst term are $\approx 20 \%$ - 30\% larger than previous results for similar energies (Birch et al., 2005; Neal et al., 2013), perhaps somewhat due to the differences in the analyses and fits (for example, the slopes of the fits to the model calculations decreased to the $0.029-0.030$ level, when $\mathrm{Kp}=8$ was also included in the fits, while the magnetic latitude offsets decrease, i.e. move equatorward). Since the $\mathrm{Kp} \leq 3$ model calculations (the three lowest Dst levels in Figure 16) deviate poleward from the linearity of the $\mathrm{Kp}=4$ to $\mathrm{Kp}=7$ region of Figure 16 , and also the mean magnetic latitude measurements don't extend to these low Dst levels, there is a slight poleward bias in the model magnetic latitude offsets compared to the measured magnetic latitude offsets presented in Table 4. The dependence of the fits on Dst seems less than for lower energy, dayside observations (Nesse TyssoY \& Stadsnes, 2015), although the dependence on Dst is similar to corresponding lower energy, nightside observations. One main advancement of these fits, at least for comparison purposes, is that we now have linear fits to the model calculations that are in reasonable agreement with the empirical fits to the measurements performed here and elsewhere (Birch et al., 2005; Neal et al., 
2013; Nesse TyssoY \& Stadsnes, 2015).

For both hemispheres, we have also compared the mean model magnetic latitudes as functions of model Dst values with the individual magnetic latitude observations as functions of measured Dst values (Figure 17). We have colored and symbol coded the observations in a manner similar to Figure 10. The general trend for the individual magnetic latitude observations to cluster along the mean model magnetic latitude curves in Figure 17 is reasonably clear. For the $\mathrm{Kp}=5$ and $\mathrm{Kp}=8$ bins, the variations of the individual measured magnetic latitudes with Dst values are fairly modest, at least compared to the intrinsic variations for these two activity levels. A careful re-examination of the Kp bins in Figure 15 also qualitatively suggests this aspect. In addition (and consistent with the Figure 16 mean magnetic latitude results), Figure 17 also shows that for $\mathrm{Kp}=8$, the individual measured magnetic latitudes for the Northern hemisphere are typically equatorward of the mean model calculations as well. This effect is more modest for the Southern hemisphere, but there are still a few Southern hemisphere, $\mathrm{Kp}=8$ observations that are significantly equatorward of the mean model magnetic latitudes. In any case, these $\mathrm{Kp}=8$ results from Figures 16 and 17 may indicate that averaging the mean model magnetic latitudes for the Dst values of the data with the original $\mathrm{Kp}=8$, Dst=-300 nT calculations would better describe these high geomagnetic activity bin observations, although somewhat different linear combinations of these two versions of the mean model calculations would be preferable for each hemisphere.

At this point, we return briefly to the results concerning at what Dst values in hypothetical model calculations would the mean magnetic latitude 
differences in Figure 15 be equal to zero. We note that for the $\mathrm{Kp}=8$ bin, Figure 17 illustrates that for the Dst $\leq-200 \mathrm{nT}$ geomagnetic disturbances, the measured magnetic latitudes have modest systematic variation with Dst. This implies that one component of the approximately $40 \mathrm{nT}$ mean Dst value differences for the $\mathrm{Kp}=8$ bin may be due to the details how the Dst values are sampled in this work, since such samplings can affect the measured mean Dst values significantly while only having a modest affect on the measured mean magnetic latitudes.

Transitioning back to the Northern hemisphere Figure 17 results from two paragraphs ago, we see that for all other $\mathrm{Kp}$ bins except the $\mathrm{Kp}=8$ bin, it appears using mean model magnetic latitude calculations at the Dst values of the data would improve the model's accuracy. For the Kp $<8$ bins of the Southern hemisphere, the analogous calculations at the measured Dst values offer conservative estimates of the mean magnetic latitudes, i.e. these mean model calculations are equatorward of the mean measured magnetic latitudes, while the Kp binned mean magnetic latitudes tend to be poleward of the mean measurements. In the context of the Southern hemisphere mean model calculations presented here, it would be possible to further improve the accuracy of the mean model magnetic latitude calculations by linear combinations of the two versions of the mean model calculations as well. Caution is warranted, however, in understanding contributing factors, such as the origin of the poleward shift of the Southern hemisphere mean measured magnetic latitudes relative to the Northern hemisphere measurements, as well as any potential differences in the penumbral effects between the two hemispheres. 


\section{Conclusion}

For the large SEP events of October 1989, we have presented NOAA10/GOES-7 measurements of 36-80 MeV proton threshold geomagnetic transmission geographic latitudes and magnetic latitudes. We have compared the results with model calculations employing the combined IGRF and Kp/Dst modified 1989 Tsyganenko models. We have shown time lines of these measured and calculated geographic and magnetic latitudes in Figures 2 - 9, as well as differences between the measured and modeled time lines, illustrating general good agreement between the measurements and Kp/Dst modeling and forming the center from which subsequent analyses were performed. We have provided Kp binned correlation plots of measured and modeled magnetic latitudes for both the Northern and Southern hemispheres in Figure 10, both further demonstrating and simplifying the relationship between the data and modeling as a function of Kp. These correlation plots also showed that the range of magnetic latitude variations was $\sim 10^{\circ}$ for the geomagnetic activity levels presented here.

We have histogrammed the differences between measured and calculated geographic and magnetic latitudes in Figures 11 and 12, both for the combination of all Kp geomagnetic activity levels and for the eight Kp bins presented here. We have included conceptually similar histograms of the differences in polar pass durations in Figures 13 and 14. We have also summarized several aspects of the analyses discussed so far in the previous section by tabulating the overall and Kp binned mean magnetic latitudes, mean differences in both geographic latitudes and magnetic latitudes, and standard deviations of the differences in magnetic latitudes in Table 2. We have also 
tabulated the differences in the mean magnetic latitudes for the Northern and Southern hemispheres separately. Similarly, we have tabluated the overall and Kp binned mean polar pass durations and mean differences in polar pass durations in Table 3.

We have found that the overall mean geographic and magnetic latitudes are around $0.45^{\circ}$ equatorward of the overall mean model calculations, with the standard deviation of the overall differences in magnetic latitudes of approximately $1.5^{\circ}$. We also have determined that as a function of the $\mathrm{Kp}$ geomagnetic activity level, the mean measured magnetic latitudes systematically vary from about $1.45^{\circ}$ equatorward of the mean calculation for the $\mathrm{Kp}=1$ bin to being around $1.0^{\circ}-1.4^{\circ}$ poleward of the mean model results for the $\mathrm{Kp}=7$ and $\mathrm{Kp}=8$ bins. Furthermore, the standard deviations of the differences in magnetic latitudes systematically increase with geomagnetic activity level. The corresponding, tabulated polar pass duration results indicated that the measured polar pass durations range from being about 0.85 minutes longer than the model results for the $\mathrm{Kp}=1$ geomagnetic activity level to being up to around 1.1 minutes shorter for the $\mathrm{Kp}=7$ geomagnetic activity level.

The two hemispheres showed somewhat different systematic variations in mean magnetic latitude differences, where the Northern hemisphere mean measured magnetic latitudes ranged from being about $1.65^{\circ}$ equatorward of the $\mathrm{Kp}=1$ calculation to being about $0.7^{\circ}-1.1^{\circ}$ poleward of the $\mathrm{Kp}=7$ and $\mathrm{Kp}=8$ calculations. For the Southern hemisphere, the mean measured magnetic latitudes ranged from being about $1.2^{\circ}$ equatorward of the $\mathrm{Kp}=1$ calculation to being about $1.25^{\circ}-1.7^{\circ}$ poleward of the $\mathrm{Kp}=7$ and $\mathrm{Kp}=8$ 
calculations.

For both hemispheres, we have presented differences in magnetic latitudes as a function of measured Dst geomagnetic activity levels for the eight $\mathrm{Kp}$ bins in Figure 15. These results indicated the general systematic variation of the differences in magnetic latitudes with Dst values, as well as an intrinisic variation, even in regions of these plots in which both $\mathrm{Kp}$ and Dst were reasonably matched. The systematic variations in Figure 15 helped motivate our efforts to improve the comparisons between the measured and model magnetic latitudes by explicitly accounting for the Dst variations.

We note that the modified 1989 Tsyganenko model calculations as developed in our earlier works (Boberg et al., 1995; Smart et al., 2006) and presented here have unique model Dst values corresponding to each Kp bin. In Figure 16, we have compared the mean model magnetic latitudes at the model Dst values with the mean measured magnetic latitudes at the mean measured Dst values. We also have included five other comparisons in Figure 16, which were as follows: the mean model magnetic latitudes interpolated to the mean measured Dst values, the mean measured magnetic latitudes for the Northern and Southern hemispheres respectively, and two curves illustrating the mean model calculations as functions of Dst for both the Northern and Southern Hemispheres. For both hemispheres, we also have compared the indivdual measured magnetic latitude results with the mean model Dst variations in Figure 17, which also showed the clustering of the mean measured magnetic latitudes along the mean model Dst curves, especially for the $\mathrm{Kp}<8$ bins. These clusterings are particularly impressive in the Northern hemisphere. Additionally, we have presented linear fits (with constant and 
linear Dst term) in Table 4 for both the mean measurements and mean model calculations (employing model Dst values from the original Kp/Dst modeling in this latter case) for the $\mathrm{Kp}<8$ bins, which have been compared with conceptually similar fits to individual measurements (not means) published elsewhere.

We have found excellent agreement between the interpolated Northern hemisphere mean model magnetic latitude results and the Northern hemisphere mean magnetic latitude and individual magnetic latitude measurements, with the potential for further improving the $\mathrm{Kp}=8$ agreement beween the interpolated mean model and mean measurement results by an averaging or other linear combination of the interpolated mean model results with the original mean $\mathrm{Kp}=8$ calculations. Figure 16 also showed that the interpolated mean model magnetic latitude, Southern hemisphere results are around $0.2^{\circ}$ to $1^{\circ}$ equatorward of the corresponding mean measured magnetic latitude results, indicating that the Southern hemisphere model interpolations provide a somewhat conservative estimate of the observed mean magnetic latitudes, while removing most of the systematic geomagnetic activity dependence of the Southern hemisphere Kp binned mean magnetic latitude differences presented in Table 2 and summarized earlier in this manuscript.

Acknowledgments: We thank D. Evans, S. Greer, R. Zwickl, and the National Geophysical Data Center for providing the NOAA-10 and GOES-7 data. The NOAA-10 orbital elements were provided by the Space Analysis and Data Branch of the US Space Command. We also thank Bill Dietrich for providing the IMP-8 sectored proton fluxes. This work was funded, in part, by the Office of Naval Research (ONR) and the NASA LWS DATM 
program under DPR W19,990. The trajectory tracings employed in this work were calculated using the DOD's Maui High Performance Computer Center (MHPCC).

\section{References}

Aschwanden, M.J., GeV particle acceleration in solar flares and Ground Level Enhancement (GLE) events, Space Sci. Rev., 171, 3-21, 2012.

Birch, M.J., Hargreaves, J.K., Senior, A., \& Bromage, B.J.I., Variations in cutoff latitude during selected solar energetic proton events, J. Geophys. Res., 110, A07221, doi:10.1029/2004/JA010833, 2005.

Boberg, P.R., Tylka, A.J., Adams, Jr., J.H., Flueckiger, E.O., \& Kobel, E., Geomagnetic transmission of solar energetic protons during the geomagnetic disturbances of October 1989, Geophys. Res. Lett., 22(9), 1133-1136, 1995.

Cliverd, M.A., Rodger, C.J., Moffat-Griffin, T., \& Verronen, P.T., Improved dynamic geomagnetic rigidity cutoff modeling: Testing predictive accuracy, J. of Geophys. Res., 112, A08302, doi:10.1029/2007JA012410, 2007.

Flueckiger, E.O., Kobel, E., Smart, D.F., \& Shea, M.A., A new concept for the simulation and visualization of cosmic ray particle transport in the Earth's magnetosphere, Proc. 22nd Int. Cosmic Ray Conf. (Dublin), 8, 648-651, 1991.

Gopalswamy, N., Xie, H., Yashiro, S., Akiyama, S., Makela, P., \& Usoskin, 
I. G., Properties of ground level enhancement events and the associated solar eruptions during solar cycle 23, Space Sci. Rev., 171, 23-60, 2012.

Kahler, S.W., Cliver, E.W., Tylka, A.J., \& Dietrich, W.,F., A comparison of ground level event $\mathrm{e} / \mathrm{p}$ and $\mathrm{Fe} / \mathrm{O}$ ratios with associated solar flare and CME characteristics, Space Sci. Rev., 171, 121-139, 2012.

Klecker, B., Popecki, M.A., Moebius, E., Desai, M.I., Mason, G.M., \& Wimmer-Schweingruber, R.F., On the energy dependence of ionic charge states, Proc. 28th Int. Cosmic Ray Conf. (Tsukuba), 6, 3277-3280, 2003.

Kress, B.T., Mertens, C.J., \& Wiltberger, M., Solar energetic particle cutoff variations during the 29-31 October 2003 geomagnetic storm, Space Weather, 8, S05001, doi:10.1029/2009SW000408, 2010.

Kress, B.T., Rodriguez, J.V., Mazur, J.E., \& Engel, M., Modeling solar proton access to geostationary spacecraft with geomagnetic cutoffs, Adv. Space Res., 52(11), 1939-1948, doi:10.10106/j.asr.2013.08.019, 2013.

Kyoto World Data Center, http://wdc.kugi.kyoto-u.ac.jp/wdc/expdata.html (See explanation of ASY index PDF, as well as the list of papers using geomagnetic indices), 2015.

Labrador, A.W., Leske, R.A., Mewaldt, R.A., Stone, E.C., \& Von Rosenvinge, T.T., High energy ionic charge state composition in recent large solar energetic particle events, Proc. 28th Int. Cosmic Ray Conf. (Tsukuba), 6, 3269-3272, 2003.

Labrador, A.W., Leske, R.A., Mewaldt, R.A., Stone, E.C., Von Rosenvinge, T.T., High energy ionic charge state composition in the October/November 
2003 and January 20, 2005 SEP events, Proc. 29th Int. Cosmic Ray Conf. (Pune), 1, 99-102, 2005.

Langel, R.A., International Geomagnetic Reference Field, 1991 Revision, J. Geomag. Geoelectr., 43, 1007-1012, 1991.

Leske, R.A., Cummings, J.R., Mewaldt, R.A., Stone, E.C., \& Von Rosenvinge, T.T., Measurements of the ionic charge states of solar energetic particles using the geomagnetic field, Astrophys. J. Letters, 452, L149-L152, doi: 10.1086/309718, 1995.

Leske, R.A., Mewaldt, R.A., Stone, E.C., \& Von Rosenvinge, T.T., Observations of geomagnetic cutoff variations during solar energetic particle events and implications for the radiation environment at the space station, J. of Geophys. Res., 106(A12), 30011-30022, doi: 10.1029/2000JA000212, 2001.

Li, G., Moore, R., Mewaldt, R.A., Zhao, L., \& Labrador, A.W., A twinCME scenario for ground level enhancement events, Space Sci. Rev., 171, 141-160, doi: 10.1007/s11214-011-9823-7, 2012.

Mason, G.M., Mazur, J.E., Looper, M.D., \& Mewaldt, R.A., Charge state measurements of solar energetic particles observed with SAMPEX, Astrophys. J., 452, 901-911, 1995.

Mazur, J.E., \& Mason, G.M., Ionization states of heavy ions in large solar particle events: 1998-2001, EOS Trans. AGU, 82(47), Fall Meet. Suppl., SH31C-03, 2001.

McIlwain, C.E., Coordinates for mapping the distribution of trapped particles, J. Geophys. Res., 66, 3681-3691, 1961. 
Mewaldt, R.A., Looper, M.D., Cohen, C.M.S., Haggerty, D.K., Labrador, A.W., Leske, R.A., Mason, G.M., Mazur, J.E., \& Von Rosenvinge, T.T., Energy spectra, composition, and other properties of ground-level events during solar cycle 23, Space Sci. Rev., 171, 97-120, 2012.

Neal, J.J., Rodger, C.J., \& Green, J.C., Empirical determination of solar proton access to the atmosphere: Impact on polar flight paths, Space Weather, 11, 420-433, doi:10.1002/swe.20066, 2013.

Nesse TyssoY, H., Stadsnes, J.,Soraas, F., \& SoRbo, M., Variations in cutoff latitude during the January 2012 solar proton event and implication for the distribution of particle energy deposition, Geophys. Res. Lett., 40(16), 4149-4153, doi:10.10002/grl.50815, 2013.

Nesse TyssoY, H., \& Stadsnes, J., Cutoff latitude variation during solar proton events: Causes and consequences, J. Geophys. Res., 120(1), 553563, doi:10.10002/2014JA020508, 2015.

Nitta, N. V., Liu, Y., DeRosa, M.L., \& Nightingale, R.W., What are special about ground-level events?, Space Sci. Rev., 171, 61-83, 2012.

National Geophysical Data Center, Boulder CO, 1994.

National Geophysical Data Center, Boulder CO, 1995.

Oetliker, M., Klecker, B., Hovestadt, D., Mason, G.M., Mazur, J.E., Leske, R.A., Mewaldt, R.A., Blake, J., \& Looper, M.D., The ionic charge of solar energetic particles with energies of 0.3-70 MeV per nucleon, Astrophys. J., 477, 495-501, doi: 10.1086/303679, 1997. 
Press, W.H., Flannery, B.P., Teukolsky, S.A., \& Vettering, W.T., Numerical Recipes, Cambridge Univ. Press, (New York, New York, 1989).

Reames, D.V., The two sources of solar energetic particles, Space Sci. Rev., 175(1-4). 53-92, doi: 10.1007/s11214-013-9958-9, 2013.

Rodger, C.J., Cliverd, M.A., Verronen, P.T., Ulich, T., Jarvis, M.J., \& Turunen, E., Dynamic geomagnetic cutoff rigidity cutoff variations during a solar proton event, J. of Geophys. Res., 111, A04222, doi: 10.1029/2005JA011395, 2006.

Sabaka, T.J., Langel, R.A., Baldwin, R.T., \& Conrad, J.A., The geomagnetic field 1990-1995, including the large scale fields from magnetospheric sources and NASA candidate models for the 1995 IGRF revision, J. Geomag. Geoelect., 49, 157-206, 1997.

Shea, M.A., \& Smart, D.F., Space Weather and the Ground-Level Solar Proton Events of the 23rd Solar Cycle, Space Sci. Rev., 171, 161-188, 2012.

Smart, D.F., \& Shea, M.A., A study of the Effectiveness of the McIlwain Coordinates in Estimating Cosmic-Ray Vertical Cutoff Rigidities, J. of Geophys. Res., 72, 3447-3454, 1967.

Smart, D.F., Shea, M.A., Golightly, M.J., Weyland, M., \& Johnson, A.S., Evaluation of the dynamic cutoff rigidity model using dosimetry data from the STS-28 flight, Adv. Space Res., 31(4), 841-846, 2003.

Smart, D.F., \& Shea, M.A., The space developed dynamic vertical cutoff and 
its applicability to aircraft radiation dose, Adv. Space Res., 32(1), 103-108, 2003.

Smart, D.F., Shea, M.A., Tylka, A.J., \& Boberg, P.R., A geomagnetic cutoff rigidity interpolation tool: accuracy verification and application to space weather, Adv. Space Res., 37, 1206-1217, 2006.

Stoer, J., \& Bulirsch, R., Introduction to Numerical Analysis, Chapter 7, Springer-Verlag, (New York, New York, 1980).

Tsurutani, B.T., Gonzalez, W.D., Lakhina, G.S., \& Alex, S., The extreme magnetic storm of 1-2 September 1859, J. Geophys. Res., 108(A7), 1268, doi:10.1029/2002JA009504, 2003.

Tsyganenko, N.A., A magnetospheric magnetic field model with a warped tail current sheet, Planet. Space Sci., 37(1), 5-20, 1989.

Tsyganenko, N.A., A model of the near magnetosphere with a dawn-dusk asymmetry: 1. Mathematical Structure, J. of Geophys. Res., 107(A8), 1179, doi:10.1029/2001JA000219, 2002a.

Tsyganenko, N.A., A model of the near magnetosphere with a dawn-dusk asymmetry: 2. Parameterization and fitting to observations, J. of Geophys. Res., 107(A8), 1176, doi:10.1029/2001JA000220, 2002b.

Tsyganenko, N.A., \& Sitnov, M.I., Modeling the dynamics of the inner magnetosphere during strong geomagnetic storms, J. of Geophys. Res., 110, A03208, doi:10.1029/2004JA010798, 2005. 
Tylka, A.J., Boberg, P.R., Adams, Jr., J.H., Beahm, L.P., Dietrich, W.F., \& Kleis, T., The mean ionic charge state of solar energetic Fe ions above 200 MeV per nucleon, Astrophys. J. Letters, 444, L109-L113, 1995.

Tylka, A.J., Adams, Jr., J.H., Boberg, P.R., Brownstein, B., Dietrich, W.F., Flueckiger, E.O., Petersen, E.L., Shea, M.A., Smart, D.F., \& Smith, E.C., CREME96: A revision of the cosmic ray effects on micro-electronics code, IEEE Trans. On Nucl. Science, 44(6), 2150-2160, 1997.

Tylka, A.J., Cohen, C.M.S., Dietrich, W.F., MacIennan, C.G., McGuire, R.E., Ng, C.K., \& Reames, D.V., Evidence for Remnant Flare Suprathermals in the source population of solar energetic particles in the 2000 Bastille Day event, Astrophys. J. Letters, 558, L59-L63, doi: 10.1086/323344, 2001.

Tylka, A.J., Cohen, C.M.S., Dietrich, W.F., Lee, M.A., MacIennan, C.G., Mewaldt, R.A., Ng, C.K., \& Reames, D.V., Shock geometry, seed populations, and the origin of variable elemental composition at high energies in large gradual solar particle events, Astrophys. J., 625, 474-495, doi: 10.1086/429384, 2005.

Wanliss, J.A., \& Showalter, K.M., High-resolution global storm index: Dst versus SYM-H, J. of Geophys. Res., 111, A02202, doi:10.1029/2005JA011034, 2006. 
1

2

3

Table 1: Definitions and equivalent terms of phrases involving the word latitude.

\begin{tabular}{|c|c|}
\hline Latitude phrase & Definition \\
\hline Geomagnetic transmission & $\begin{array}{l}\text { The ratio of the particle flux at } \\
\text { a spacecraft's location within the } \\
\text { magnetosphere to the interplane- } \\
\text { tary flux. In this work, the NOAA- } \\
\text { 10/GOES- } 7 \text { fluxes and correspond- } \\
\text { ing calculations. }\end{array}$ \\
\hline $\begin{array}{l}\text { Measured geographic latitudes, } \\
\text { measured threshold geographic } \\
\text { latitudes, measured threshold } \\
\text { geomagnetic transmission } \\
\text { geographic latitudes }\end{array}$ & $\begin{array}{l}\text { The geographic latitudes } \\
\text { corresponding to the measured } \\
\text { NOAA-10/GOES-7 geomagnetic } \\
\text { transmissions, interpolated to the } \\
\text { geomagnetic transmissions' } 10 \% \\
\text { levels. }\end{array}$ \\
\hline $\begin{array}{l}\text { Calculated geographic latitudes, } \\
\text { calculated threshold geographic } \\
\text { latitudes, calculated threshold } \\
\text { geomagnetic transmission } \\
\text { geographic latitudes }\end{array}$ & $\begin{array}{l}\text { The geographic latitudes } \\
\text { corresponding to the IGRF + } \\
\text { modified Tsyganenko } 1989 \text { model } \\
\text { calculations' NOAA-10/GOES-7 } \\
\text { geomagnetic transmissions, } \\
\text { interpolated to the geomagnetic } \\
\text { transmissions' } 10 \% \text { levels. }\end{array}$ \\
\hline $\begin{array}{l}\text { Measured (invariant) magnetic } \\
\text { latitudes, measured threshold } \\
\text { magnetic latitudes, measured } \\
\text { threshold geomagnetic } \\
\text { transmission magnetic latitudes }\end{array}$ & $\begin{array}{l}\text { The magnetic latitudes } \\
\text { corresponding to the measured } \\
\text { NOAA-10/GOES-7 geomagnetic } \\
\text { transmissions, interpolated to the } \\
\text { geomagnetic transmissions' } 10 \% \\
\text { levels. }\end{array}$ \\
\hline
\end{tabular}


Table 1: (continued) Definitions and equivalent terms of phrases involving the word latitude.

\begin{tabular}{|c|c|}
\hline Latitude phrase & Definition \\
\hline Calculated (invariant) magnetic & The magnetic latitudes \\
\hline latitudes, model magnetic & corresponding to the model \\
\hline latitudes, calculated threshold & calculations' NOAA-10/GOES-7 \\
\hline magnetic latitudes, calculated & geomagnetic transmissions, \\
\hline threshold geomagnetic & interpolated to the calculated \\
\hline transmission magnetic latitudes & geomagnetic transmissions' $10 \%$ \\
\hline & levels. \\
\hline Magnetic latitudes (no measured & This means both the measured \\
\hline or calculated adjectives) & and calculated magnetic latitudes \\
\hline & are being discussed or compared. \\
\hline \multirow[t]{9}{*}{ Mean measured magnetic latitudes } & The averages of the measured \\
\hline & magnetic latitudes over the data \\
\hline & set. The means can be for entire \\
\hline & data set, means for geomagnetic \\
\hline & activity levels, means for the \\
\hline & different hemispheres, or means for \\
\hline & combinations of these possibilities. \\
\hline & See Table 2 for examples of the \\
\hline & possible mean magnetic latitudes. \\
\hline
\end{tabular}


Table 1: (continued) Definitions and equivalent terms of phrases involving the word latitude.

\begin{tabular}{l|l}
\hline \multicolumn{1}{c}{ Latitude phrase } & \multicolumn{1}{c}{ Definition } \\
\hline Mean calculated magnetic & The mean model calculation \\
latitudes, model mean magnetic & magnetic latitudes, conceptually \\
latitudes, mean model magnetic & similar to the mean measured \\
latitudes & magnetic latitudes. \\
\hline Cutoff latitudes, cutoff magnetic & In this work, these phrases are \\
latitudes & $\begin{array}{l}\text { generally used in either the } \\
\text { keywords, or in citing other } \\
\text { published results. Our procedure } \\
\text { for calculating cutoff latitudes is } \\
\text { described in the first three } \\
\text { paragraphs of Section 3 } \\
\text { (Geogmagnetic Transmission } \\
\text { Modeling). Other works have } \\
\text { employed conceptually similar } \\
\text { methods (see those other cited } \\
\text { works for differences in the details, } \\
\text { including how other works } \\
\text { determine the measured cutoff } \\
\text { latitudes as well). }\end{array}$ \\
\hline
\end{tabular}


Table 1: (continued) Definitions and equivalent terms of phrases involving the word latitude.

\begin{tabular}{|c|c|}
\hline Latitude phrase & Definition \\
\hline Interpolated latitudes & $\begin{array}{l}\text { We perform three types of latitude } \\
\text { interpolations in this work. The } \\
\text { first is described in Section } 2 \\
\text { (Geomagnetic Transmission } \\
\text { Measurements), as well as the } \\
\text { equivalent part of Section } 3 \\
\text { (Geomagnetic Transmission } \\
\text { Modeling). This interpolation } \\
\text { corresponds to interpolations to } \\
\text { the } 10 \% \text { geomagnetic transmission } \\
\text { threshold levels of the } \\
\text { measurements and calculations. } \\
\text { The second type of interpolations } \\
\text { are employed in the conversion } \\
\text { from the geomagnetic cutoff grids } \\
\text { to the geomagnetic cutoffs at the } \\
\text { spacecraft locations (see Section } 3 \text {, } \\
\text { Geomagnetic Transmission } \\
\text { Modeling). The final, cumulative } \\
\text { type of interpolations convert from } \\
\text { the mean calculated magnetic } \\
\text { latitudes as functions of Kp } \\
\text { geomagnetic activity levels to the } \\
\text { mean Dst geomagnetic activity } \\
\text { levels of the data (see last seven } \\
\text { paraphs of Section } 4 \text {, }\end{array}$ \\
\hline
\end{tabular}




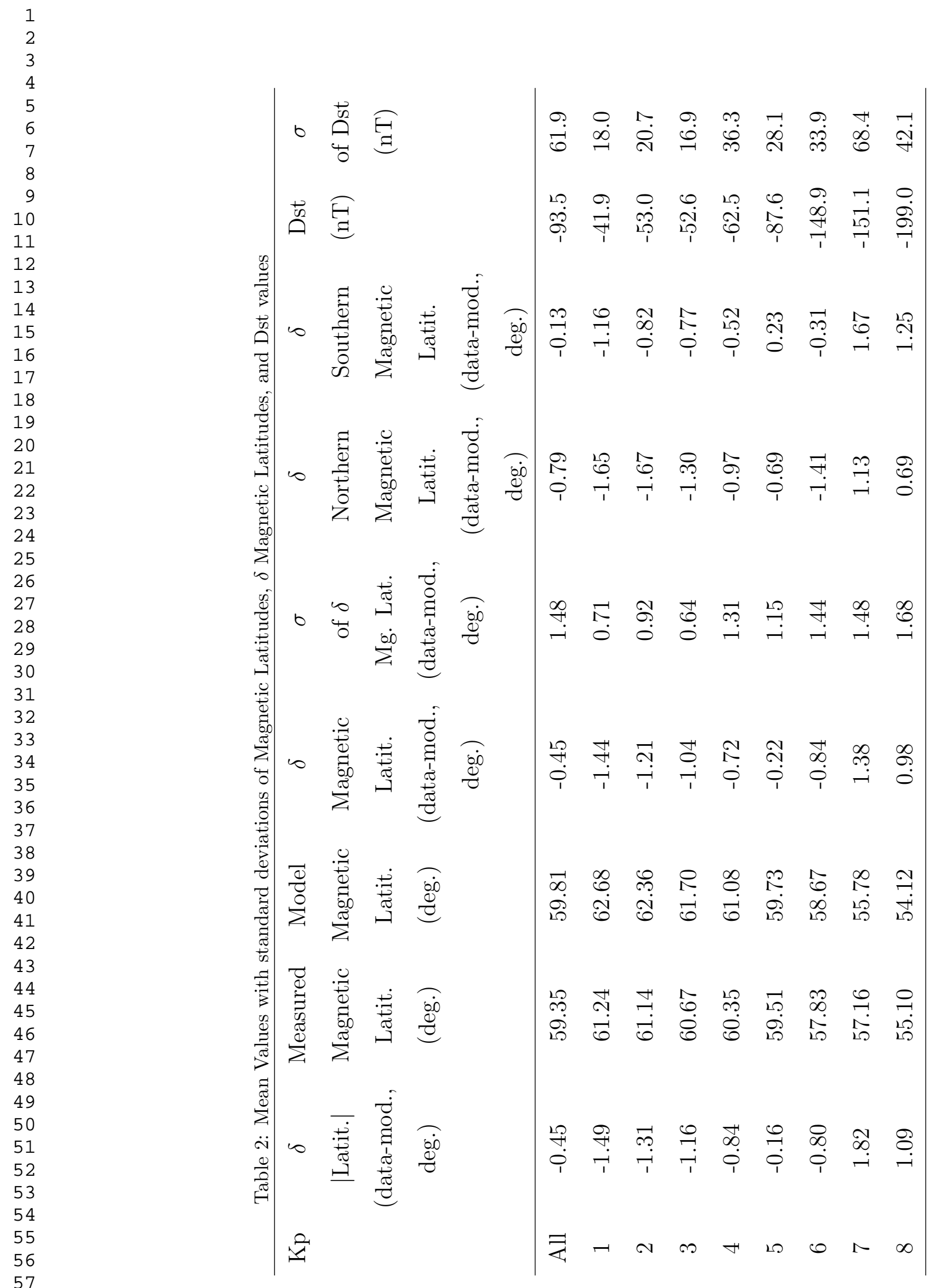


Table 3: Mean polar pass durations and differences

\begin{tabular}{|c|c|c|c|c|}
\hline \multirow[t]{6}{*}{$\mathrm{Kp}$} & Measured & Model & $\delta$ & $\sigma$ \\
\hline & Polar Pass & Polar Pass & Polar Pass & of $\delta$ \\
\hline & Duration & Duration & Duration & Polar Pass \\
\hline & (min.) & (min.) & (data-mod., & Duration \\
\hline & & & $\min )$. & (data-mod., \\
\hline & & & & min.) \\
\hline All & 17.53 & 17.26 & 0.27 & 0.96 \\
\hline 1 & 16.92 & 16.07 & 0.85 & 0.46 \\
\hline 2 & 16.90 & 16.13 & 0.77 & 0.57 \\
\hline 3 & 16.46 & 15.79 & 0.67 & 0.34 \\
\hline 4 & 16.77 & 16.33 & 0.44 & 0.84 \\
\hline 5 & 16.94 & 16.79 & 0.15 & 0.75 \\
\hline 6 & 18.34 & 17.82 & 0.51 & 0.99 \\
\hline 7 & 18.32 & 19.43 & -1.11 & 1.17 \\
\hline 8 & 20.97 & 21.59 & -0.62 & 0.88 \\
\hline
\end{tabular}

Table 4: Linear Dst fit parameters for the mean measured and modeled magnetic latitudes for $\mathrm{Kp}<8$ intervals

\begin{tabular}{ccc}
\hline Fit & Magnetic Latit. Offset & slope of Dst term \\
\hline Data (North) & $62.18 \pm 0.20$ & $0.0340 \pm 0.0021$ \\
Data (South) & $63.14 \pm 0.26$ & $0.0353 \pm 0.0027$ \\
Model (North) & $62.46 \pm 0.19$ & $0.0348 \pm 0.0021$ \\
Model (South) & $62.74 \pm 0.25$ & $0.0367 \pm 0.0028$ \\
\hline
\end{tabular}




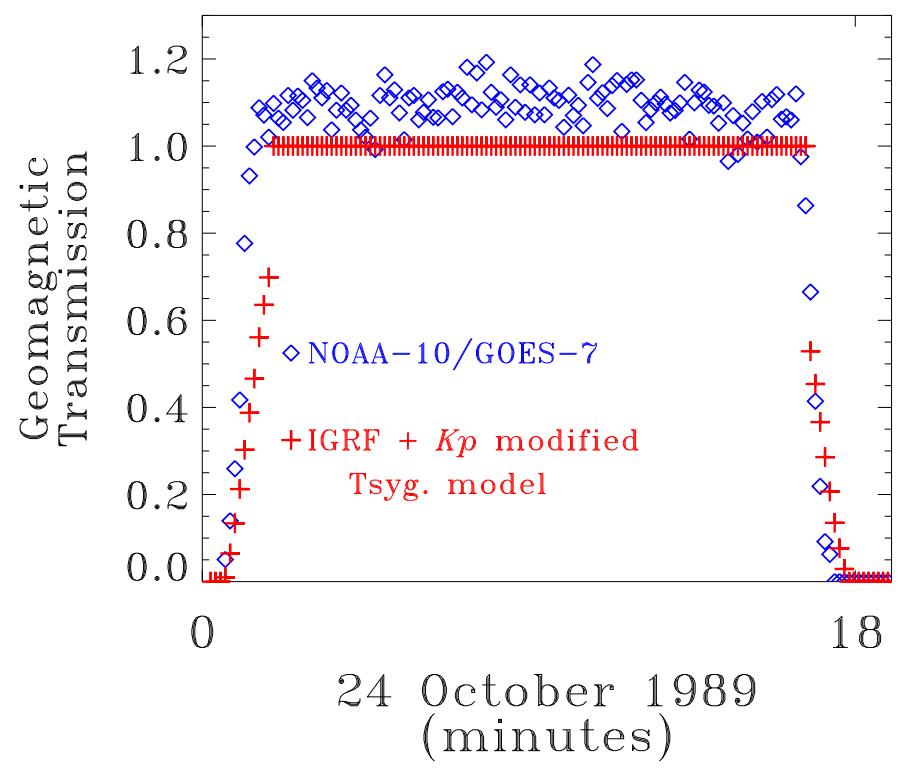

Figure 1: For an example polar pass, a comparison of the measured NOAA-10/GOES-7 geomagnetic transmission values with the corresponding IGRF + modified Tsyganenko 1989 model calculations. The sample period begins at 24 October 1989, 0 UT, and 18 minutes are shown.

Print version: For an example polar pass, a comparison of the measured NOAA-10/GOES7 geomagnetic transmission values (lighter diamonds) with the corresponding IGRF + modified Tsyganenko 1989 model calculations (darker plus signs). The sample period begins at 24 October 1989, 0 UT, and 18 minutes are shown. 
Threshold Transmission Latitudes

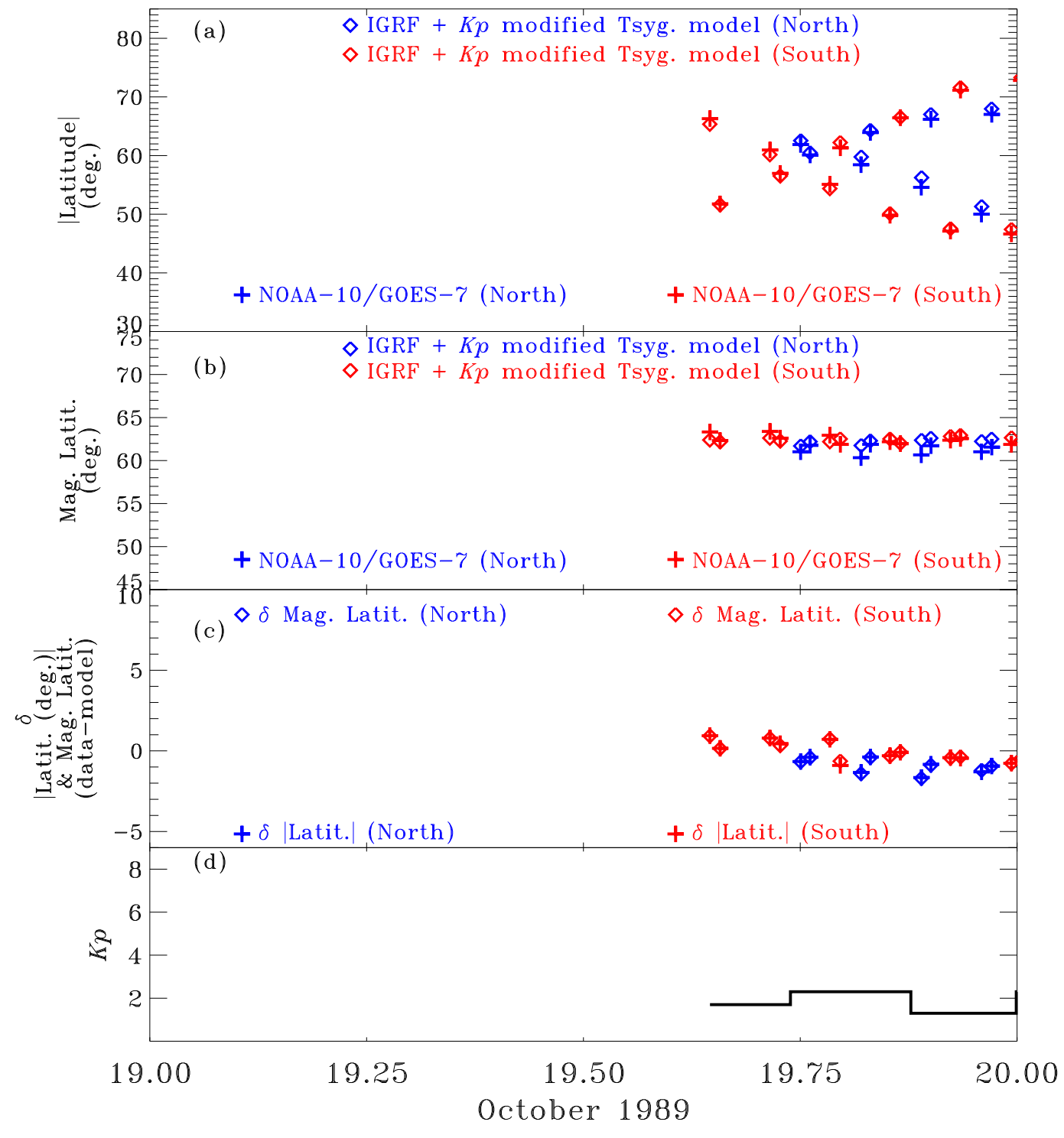


Figure 2: October 19, 1989 transmission threshold cutoff latitudes. (a) Geographic latitudes; North (blue plus signs) and South (red plus signs). The geographic latitudes are absolute values. (b) Invariant magnetic latitudes (McIlwain, 1961; Sabaka et al., 1997); North (blue plus signs) and South (red plus signs). (c) Differences between modeled and measured magnetic latitude for North (blue) and South (red). (d) Kp geomagnetic indices. (See caption for Figure 3 for a more detailed description of this figure.)

Print version: October 19, 1989 transmission threshold cutoff latitudes. (a) Geographic latitudes; North (lighter plus signs) and South (darker plus signs). The geographic latitudes are absolute values. (b) Invariant magnetic latitudes (McIlwain, 1961; Sabaka et al., 1997); North (lighter plus signs) and South (darker plus signs). (c) Differences between modeled and measured magnetic latitude for North (lighter) and South (darker). (d) Kp geomagnetic indices. (See caption for Figure 3 for a more detailed description of this figure.) 
Threshold Transmission Latitudes

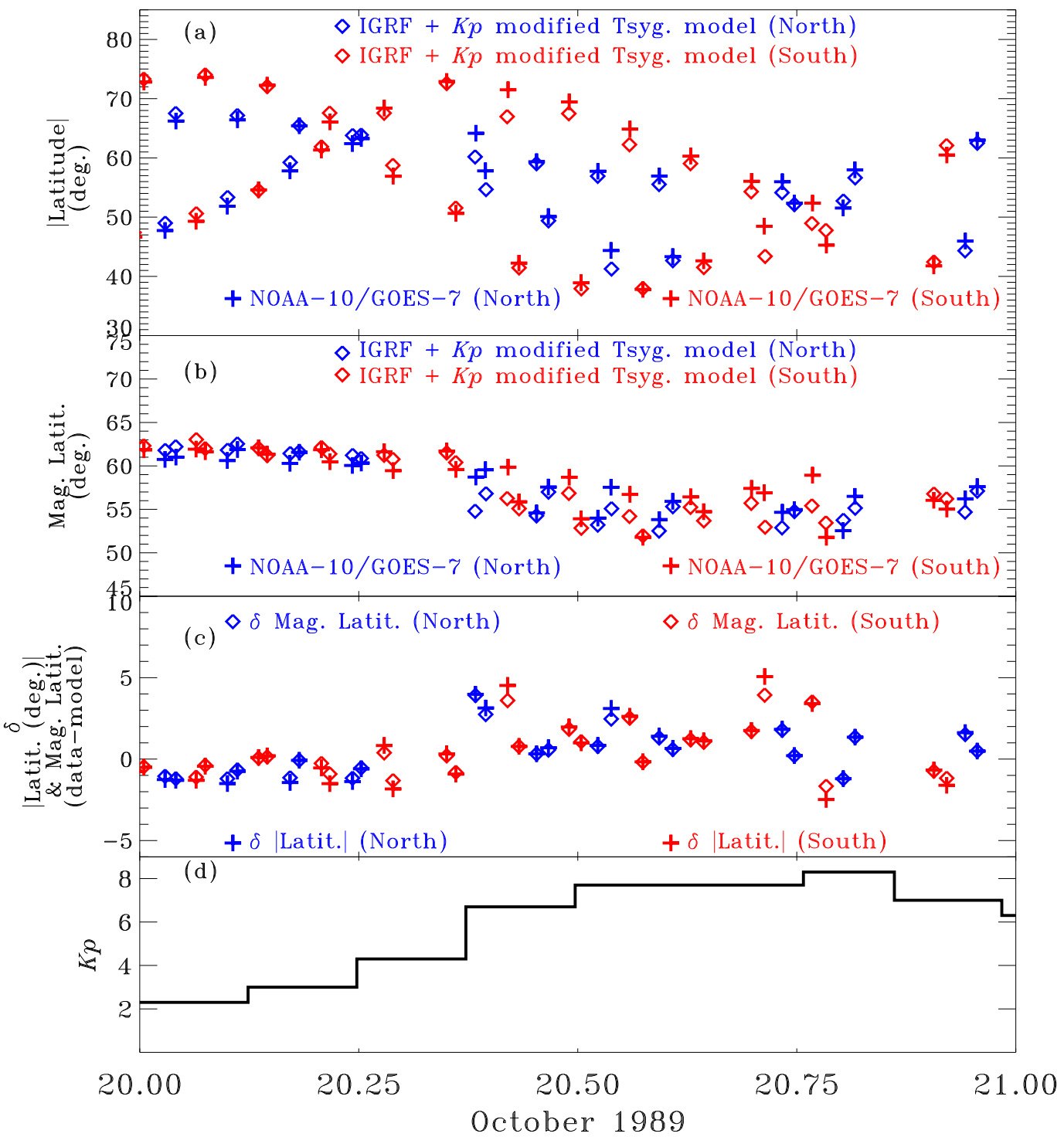


Figure 3: October 20, 1989 transmission threshold cutoff latitudes. (a) A Comparison of measured (absolute values of) geographic latitudes corresponding to NOAA-10/GOES-7 threshold geomagnetic transmission values for the Northern Hemisphere (blue plus signs) and Southern Hemisphere (red plus signs) with IGRF + modified Tsyganenko 1989 model calculations (Northern: blue diamonds; Southern red diamonds). (b) Comparisons of measured and model magnetic latitudes, using the same colors and symbols as in panel (a). (c) Differences in geographic latitudes (absolute value) between measurements and model calculations for Northern Hemisphere (blue plus signs) and Southern Hemisphere (red plus signs), as well as differences in magnetic latitudes [Northern hemisphere (blue diamonds); Southern hemisphere (red diamonds)]; and (d) Kp geomagnetic activity indices. See text for details.

Print version: October 20, 1989 transmission threshold cutoff latitudes. (a) A Comparison of measured (absolute values of) geographic latitudes corresponding to NOAA-10/GOES-7 threshold geomagnetic transmission values for the Northern Hemisphere (lighter plus signs) and Southern Hemisphere (darker plus signs) with IGRF + modified Tsyganenko 1989 model calculations (Northern: lighter diamonds; Southern darker diamonds). (b) Comparisons of measured and model magnetic latitudes, using the same shading and symbols as in panel (a). (c) Differences in geographic latitudes (absolute value) between measurements and model calculations for Northern Hemisphere (lighter plus signs) and Southern Hemisphere (darker plus signs), as well as differences in magnetic latitudes [Northern hemisphere (lighter diamonds); Southern hemisphere (darker diamonds)]; and (d) Kp geomagnetic activity indices. See text for details. 
Threshold Transmission Latitudes

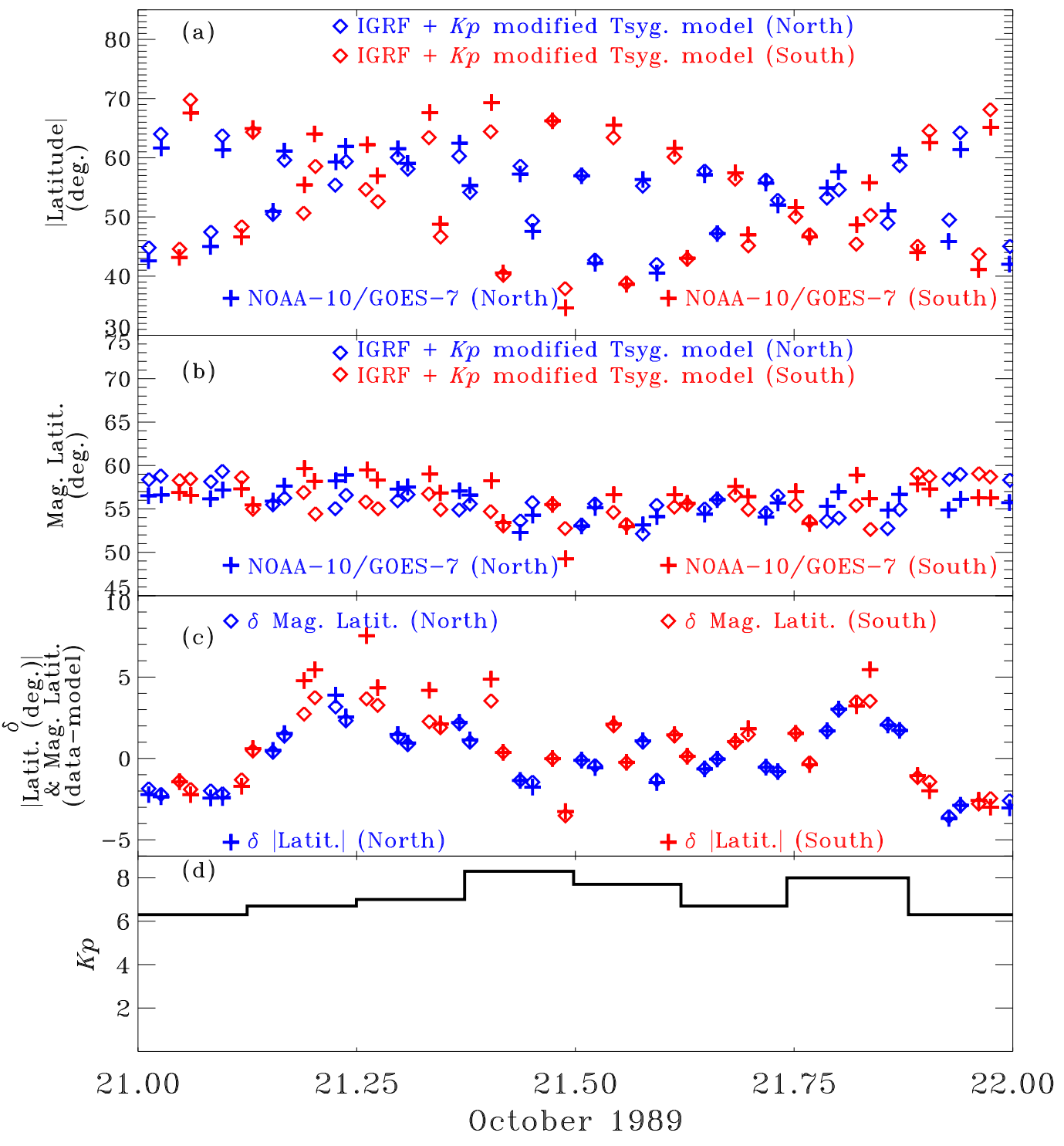


Figure 4: October 21, 1989 transmission threshold cutoff latitudes. (a) Geographic latitudes; North (blue plus signs) and South (red plus signs). The geographic latitudes are absolute values. (b) Invariant magnetic latitudes; North (blue plus signs) and South (red plus signs). (c) Differences between modeled and measured magnetic latitude for North (blue) and South (red). (d) Kp geomagnetic indices. (See caption for Figure 3 for a more detailed description of this figure.)

Print version: October 21, 1989 transmission threshold cutoff latitudes. (a) Geographic latitudes; North (lighter plus signs) and South (darker plus signs). The geographic latitudes are absolute values. (b) Invariant magnetic latitudes; North (lighter plus signs) and South (darker plus signs). (c) Differences between modeled and measured magnetic latitude for North (lighter) and South (darker). (d) Kp geomagnetic indices. (See caption for Figure 3 for a more detailed description of this figure.) 
Threshold Transmission Latitudes

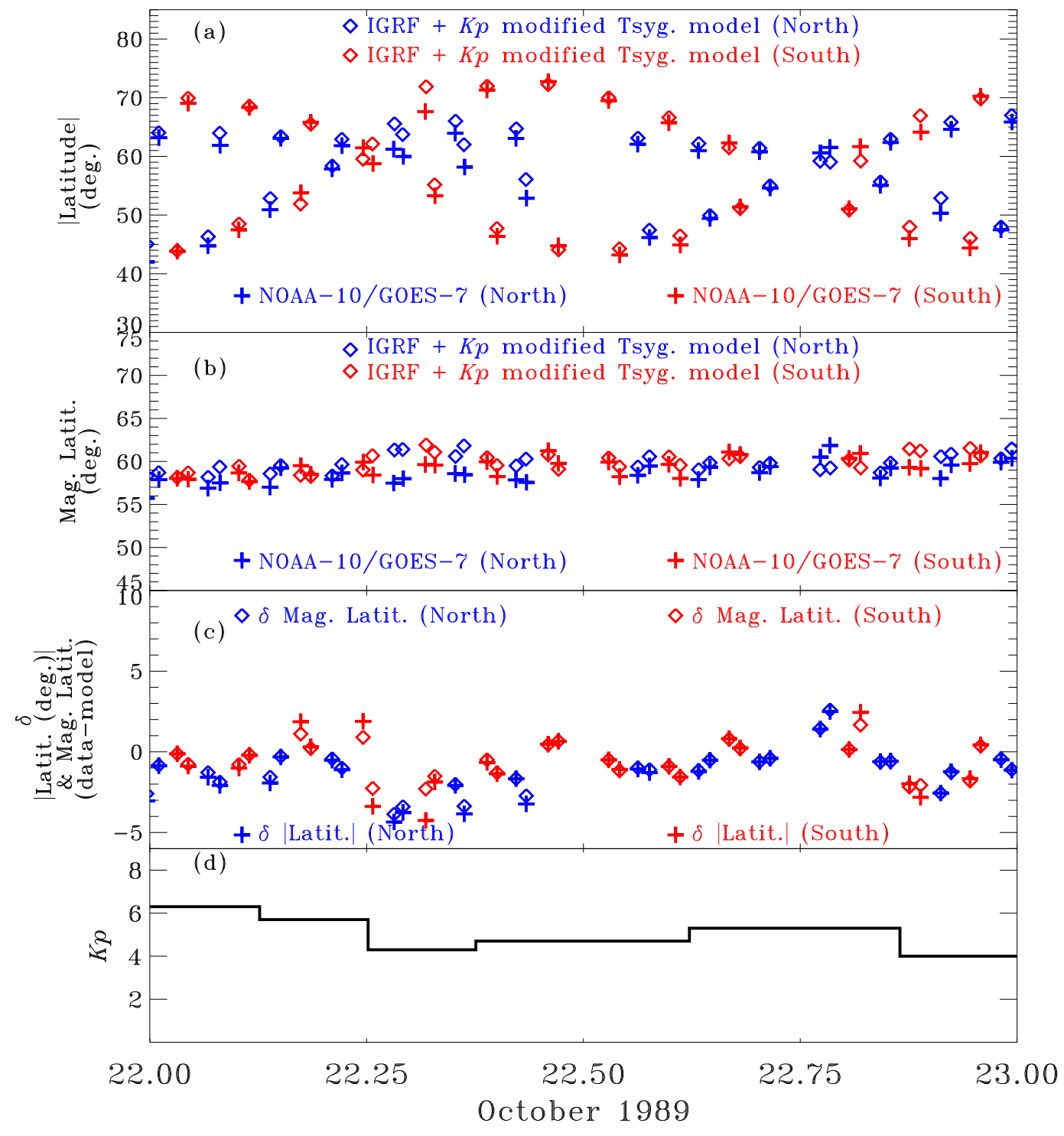

46 
Figure 5: October 22, 1989 transmission threshold cutoff latitudes. (a) Geographic latitudes; North (blue plus signs) and South (red plus signs). The geographic latitudes are absolute values. (b) Invariant magnetic latitudes; North (blue plus signs) and South (red plus signs). (c) Differences between modeled and measured magnetic latitude for North (blue) and South (red). (d) Kp geomagnetic indices. (See caption for Figure 3 for a more detailed description of this figure.)

Print version: October 22, 1989 transmission threshold cutoff latitudes. (a) Geographic latitudes; North (lighter plus signs) and South (darker plus signs). The geographic latitudes are absolute values. (b) Invariant magnetic latitudes; North (lighter plus signs) and South (darker plus signs). (c) Differences between modeled and measured magnetic latitude for North (lighter) and South (darker). (d) Kp geomagnetic indices. (See caption for Figure 3 for a more detailed description of this figure.) 
8

Threshold Transmission Latitudes

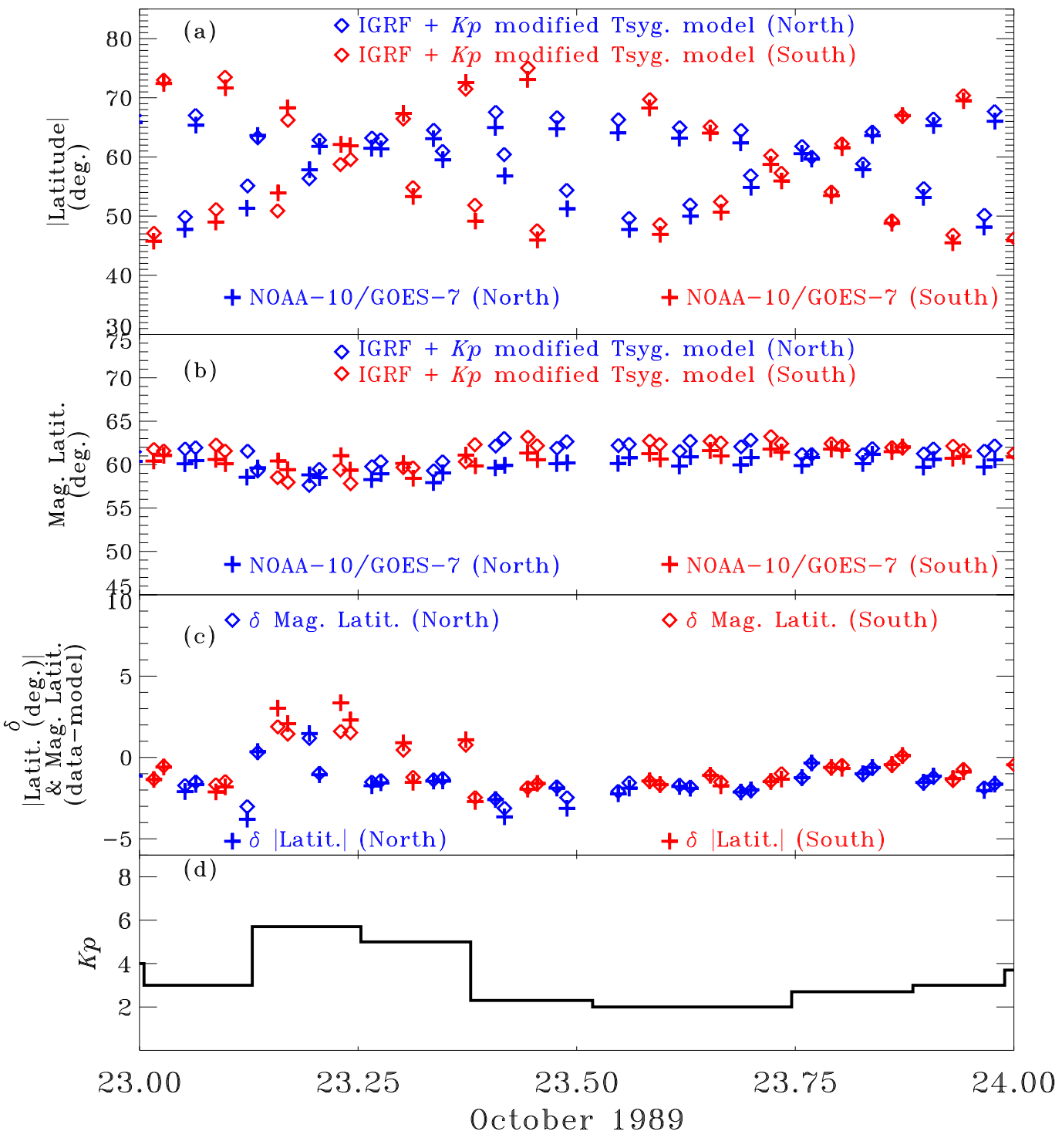


Figure 6: October 23, 1989 transmission threshold cutoff latitudes. (a) Geographic latitudes; North (blue plus signs) and South (red plus signs). The geographic latitudes are absolute values. (b) Invariant magnetic latitudes; North (blue plus signs) and South (red plus signs). (c) Differences between modeled and measured magnetic latitude for North (blue) and South (red). (d) Kp geomagnetic indices. (See caption for Figure 3 for a more detailed description of this figure.)

Print version: October 23, 1989 transmission threshold cutoff latitudes. (a) Geographic latitudes; North (lighter plus signs) and South (darker plus signs). The geographic latitudes are absolute values. (b) Invariant magnetic latitudes; North (lighter plus signs) and South (darker plus signs). (c) Differences between modeled and measured magnetic latitude for North (lighter) and South (darker). (d) Kp geomagnetic indices. (See caption for Figure 3 for a more detailed description of this figure.) 
Threshold Transmission Latitudes

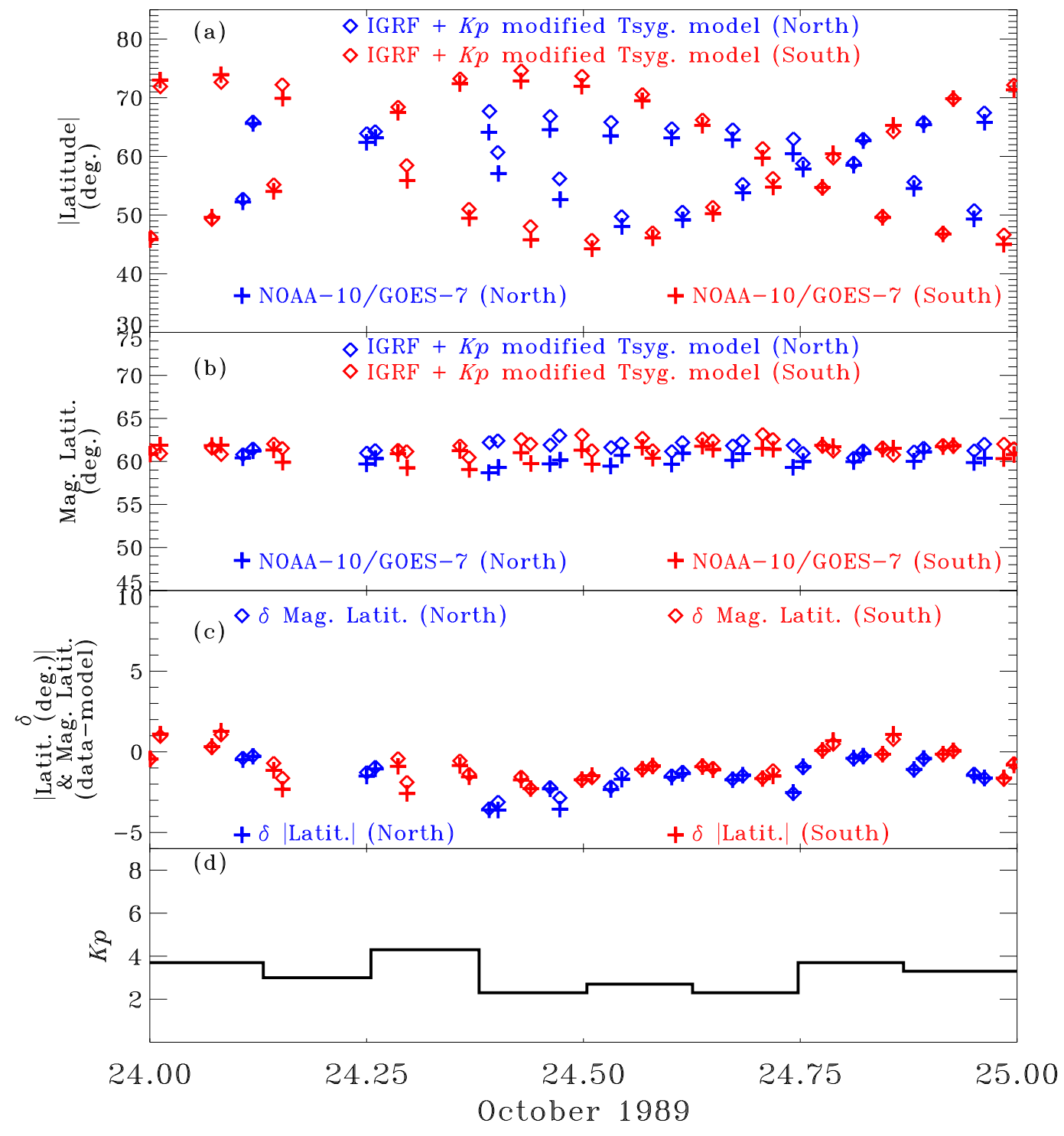


Figure 7: October 24, 1989 transmission threshold cutoff latitudes. (a) Geographic latitudes; North (blue plus signs) and South (red plus signs). The geographic latitudes are absolute values. (b) Invariant magnetic latitudes; North (blue plus signs) and South (red plus signs). (c) Differences between modeled and measured magnetic latitude for North (blue) and South (red). (d) Kp geomagnetic indices. (See caption for Figure 3 for a more detailed description of this figure.)

Print version: October 24, 1989 transmission threshold cutoff latitudes. (a) Geographic latitudes; North (lighter plus signs) and South (darker plus signs). The geographic latitudes are absolute values. (b) Invariant magnetic latitudes; North (lighter plus signs) and South (darker plus signs). (c) Differences between modeled and measured magnetic latitude for North (lighter) and South (darker). (d) Kp geomagnetic indices. (See caption for Figure 3 for a more detailed description of this figure.) 
Threshold Transmission Latitudes 
Figure 8: October 25, 1989 transmission threshold cutoff latitudes. (a) Geographic latitudes; North (blue plus signs) and South (red plus signs). The geographic latitudes are absolute values. (b) Invariant magnetic latitudes; North (blue plus signs) and South (red plus signs). (c) Differences between modeled and measured magnetic latitude for North (blue) and South (red). (d) Kp geomagnetic indices. (See caption for Figure 3 for a more detailed description of this figure.)

Print version: October 25, 1989 transmission threshold cutoff latitudes. (a) Geographic latitudes; North (lighter plus signs) and South (darker plus signs). The geographic latitudes are absolute values. (b) Invariant magnetic latitudes; North (lighter plus signs) and South (darker plus signs). (c) Differences between modeled and measured magnetic latitude for North (lighter) and South (darker). (d) Kp geomagnetic indices. (See caption for Figure 3 for a more detailed description of this figure.) 
Threshold Transmission Latitudes

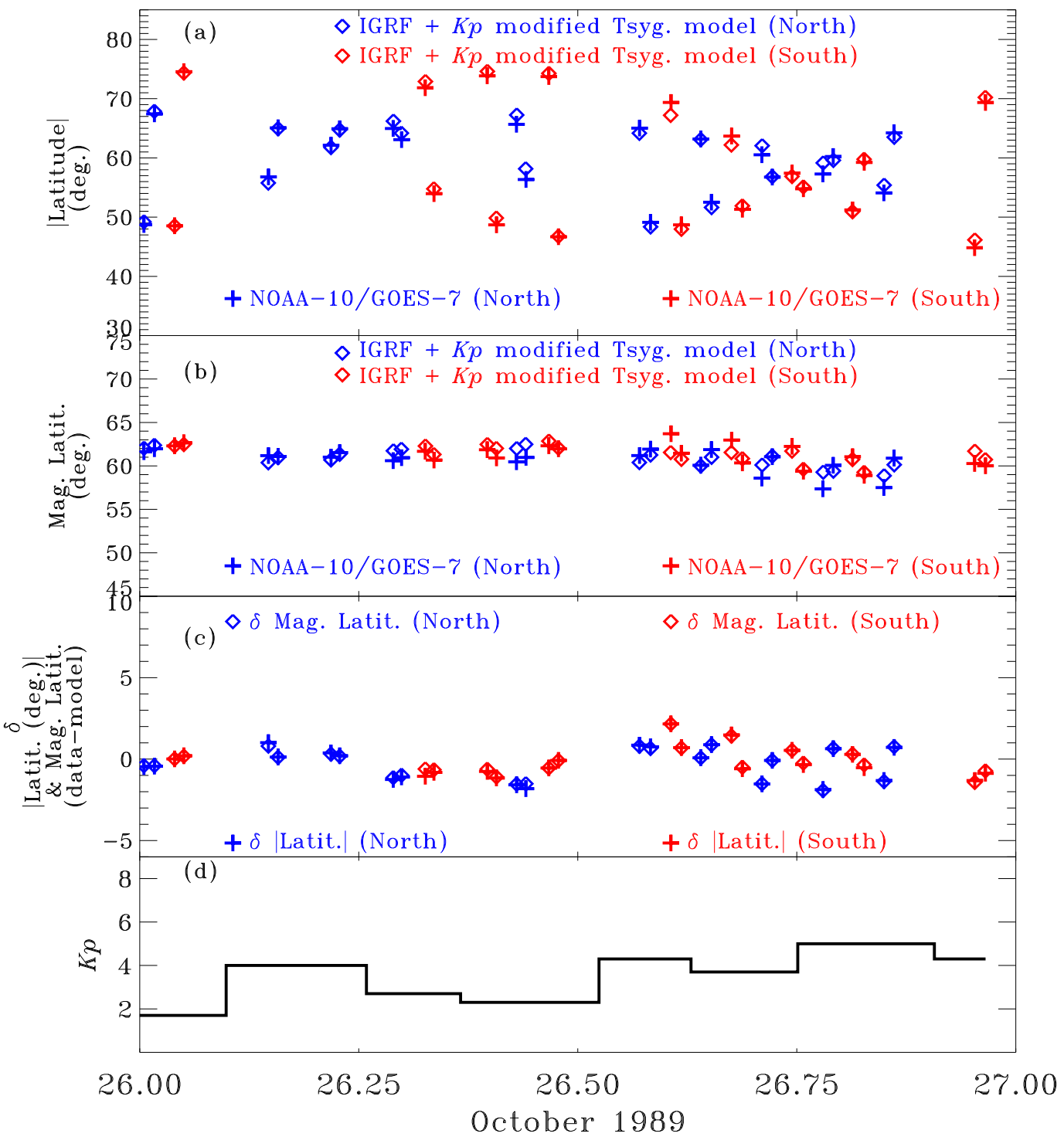


Figure 9: October 26, 1989 transmission threshold cutoff latitudes. (a) Geographic latitudes; North (blue plus signs) and South (red plus signs). The geographic latitudes are absolute values. (b) Invariant magnetic latitudes; North (blue plus signs) and South (red plus signs). (c) Differences between modeled and measured magnetic latitude for North (blue) and South (red). (d) Kp geomagnetic indices. (See caption for Figure 3 for a more detailed description of this figure.)

Print version: October 26, 1989 transmission threshold cutoff latitudes. (a) Geographic latitudes; North (lighter plus signs) and South (darker plus signs). The geographic latitudes are absolute values. (b) Invariant magnetic latitudes; North (lighter plus signs) and South (darker plus signs). (c) Differences between modeled and measured magnetic latitude for North (lighter) and South (darker). (d) Kp geomagnetic indices. (See caption for Figure 3 for a more detailed description of this figure.) 

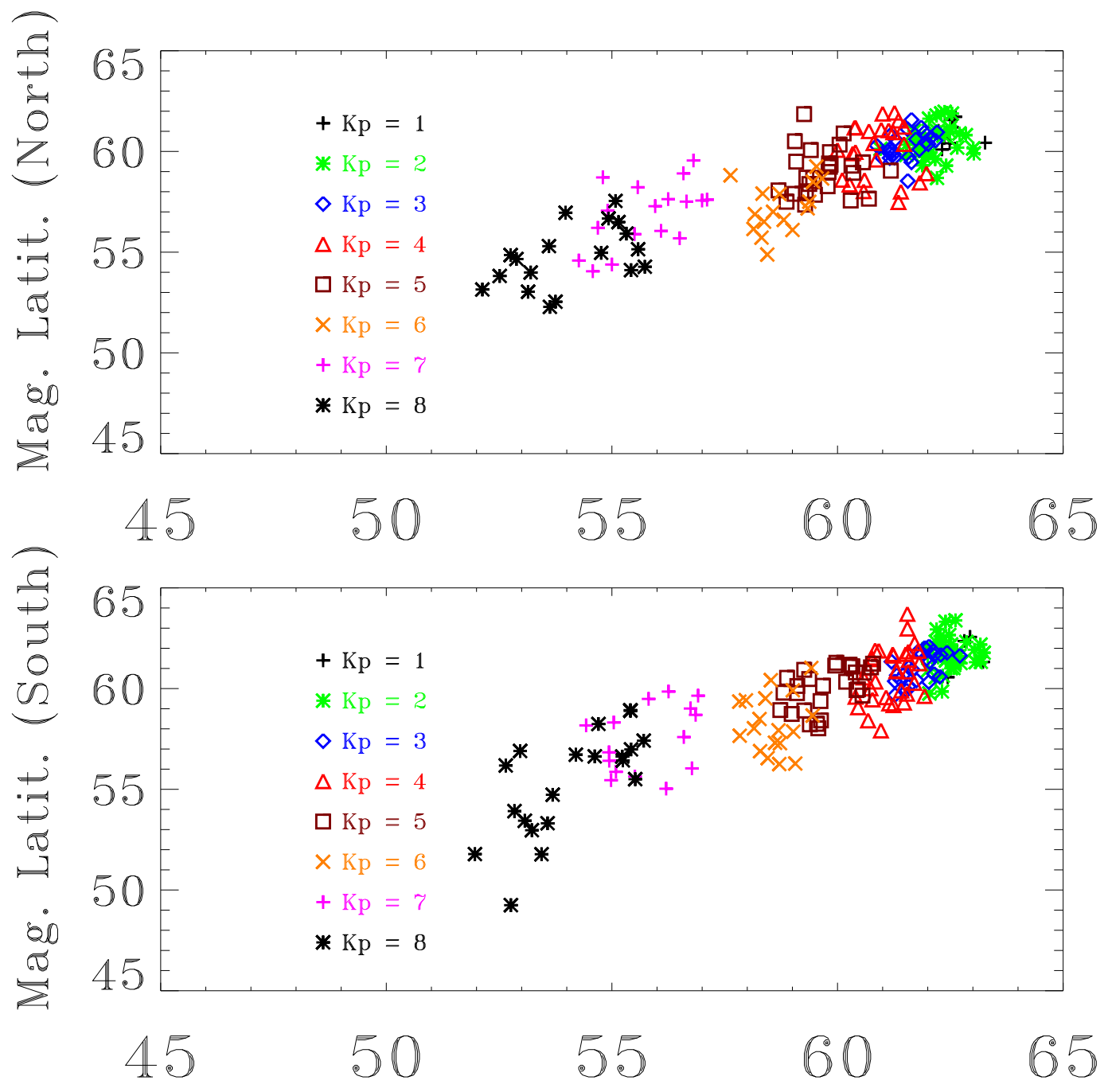
calc. Mag. Latit. 
Figure 10: Measured magnetic latitudes versus calculated magnetic latitudes, color-coded by the eight Kp levels observed during these SEP events (upper - Northern Hemisphere, lower - Southern Hemisphere).

Print version: Measured magnetic latitudes versus calculated magnetic latitudes, shaded and symbol-coded by the eight Kp levels observed during these SEP events (upper Northern Hemisphere, lower - Southern Hemisphere). 
Figure 11: Histogram of differences of geographic latitudes (data minus combined IGRF and modified 1989 Tsyganenko model), solid blue, and of differences in magnetic latitudes (data minus combined IGRF and modified 1989 Tsyganenko model), red.

Print version: Histogram of differences of geographic latitudes (data minus combined IGRF and modified 1989 Tsyganenko model), solid, lighter shading, and of differences in magnetic latitudes (data minus combined IGRF and modified 1989 Tsyganenko model), darker shading. 
Kp binned histograms of Counts vs. $\delta$ Latitudes
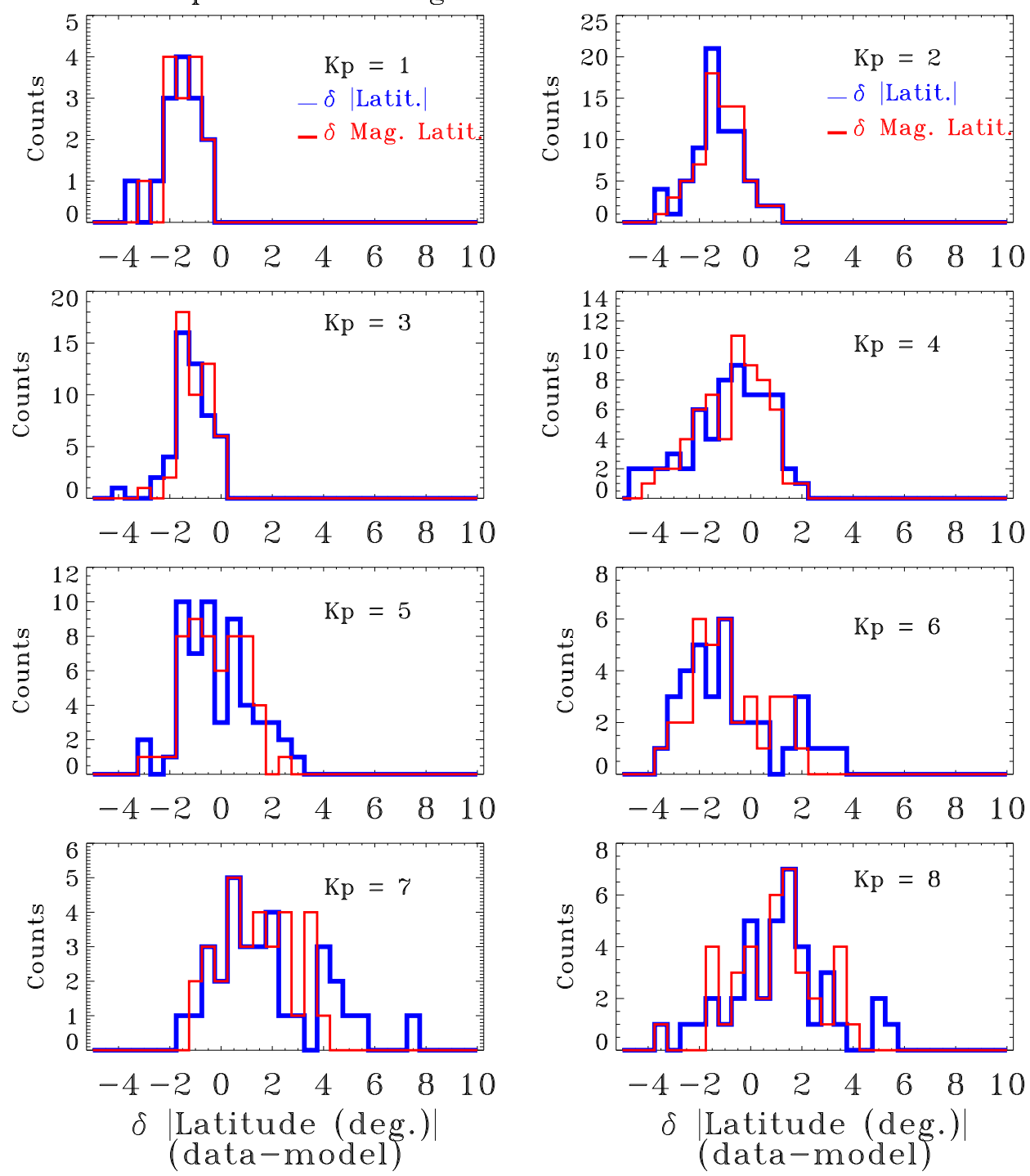

Figure 12: Kp binned histograms of differences in geographic latitudes (data minus combined IGRF and modified 1989 Tsyganenko model), solid blue; and of differences in magnetic latitudes (data minus combined IGRF and modified 1989 Tsyganenko model), red. Print version: Kp binned histograms of differences in geographic latitudes (data minus combined IGRF and modified 1989 Tsyganenko model), solid, lighter, thicker shading; and of differences in magnetic latitudes (data minus combined IGRF and modified 1989 Tsyganenko model), darker, thinner shading. 


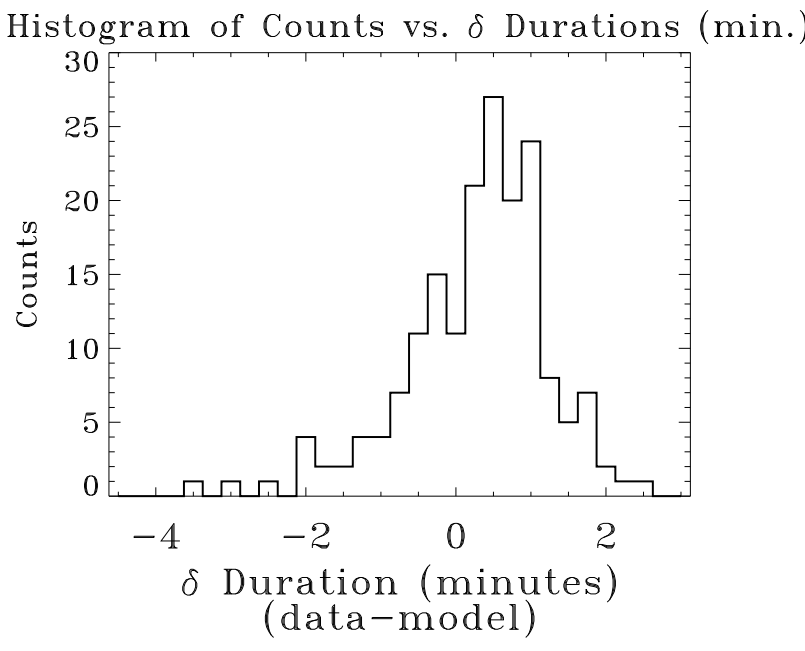

Figure 13: Histogram of differences in durations of polar passes in minutes, (data minus combined IGRF and modified 1989 Tsyganenko model).

Print version: Histogram of differences in durations of polar passes in minutes, (data minus combined IGRF and modified 1989 Tsyganenko model). 
Kp binned histograms of Counts vs. $\delta$ Durations (minutes)
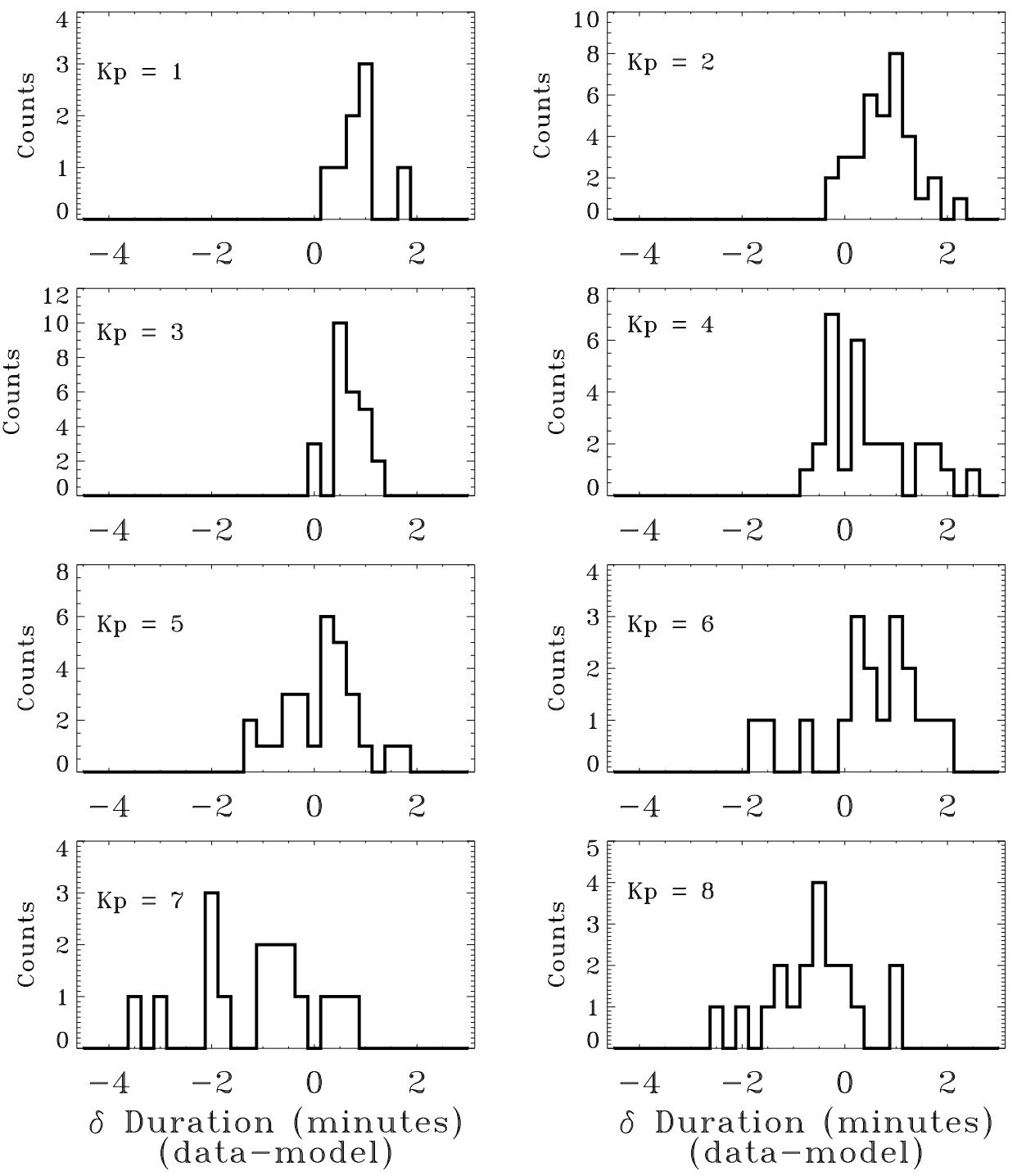
Figure 14: Kp binned histograms of differences in durations of polar passes in minutes, (data minus combined IGRF and modified 1989 Tsyganenko model). Print version: Kp binned histograms of differences in durations of polar passes in minutes, (data minus combined IGRF and modified 1989 Tsyganenko model). 

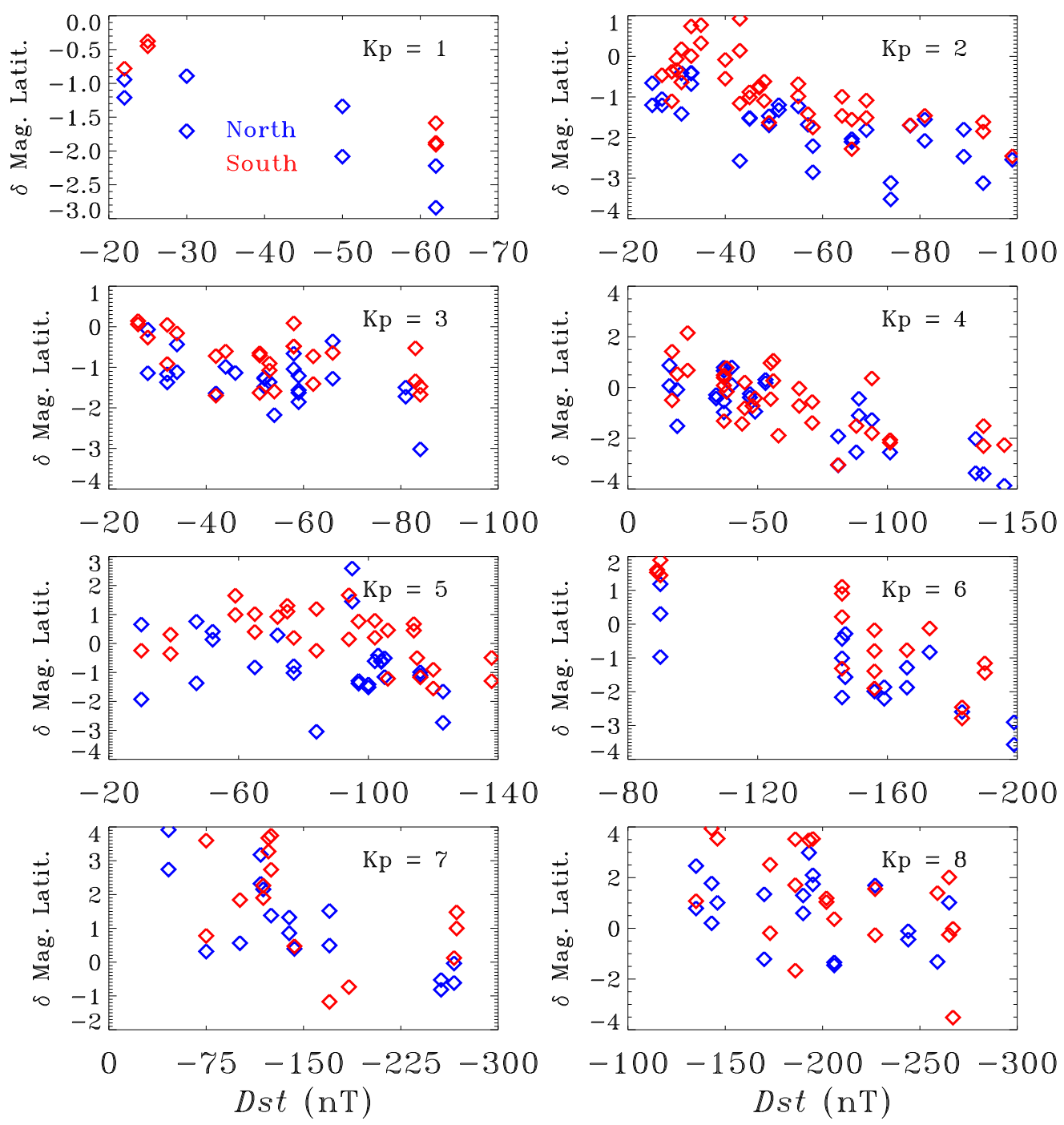

Figure 15: For eight Kp bins, plots of differences in magnetic latitudes (data minus combined IGRF and modified 1989 Tsyganenko model) versus the measured Dst values. The Northern hemisphere passes are shown in blue, and the Southern hemisphere passes are shown in red.

Print version: For eight Kp bins, plots of differences in magnetic latitudes (data minus combined IGRF and modified 1989 Tsyganenko model) versus the measured Dst values. The Northern hemisphere passes are shown in lighter diamonds, and the Southern hemisphere passes are shown in darker diamonds. 


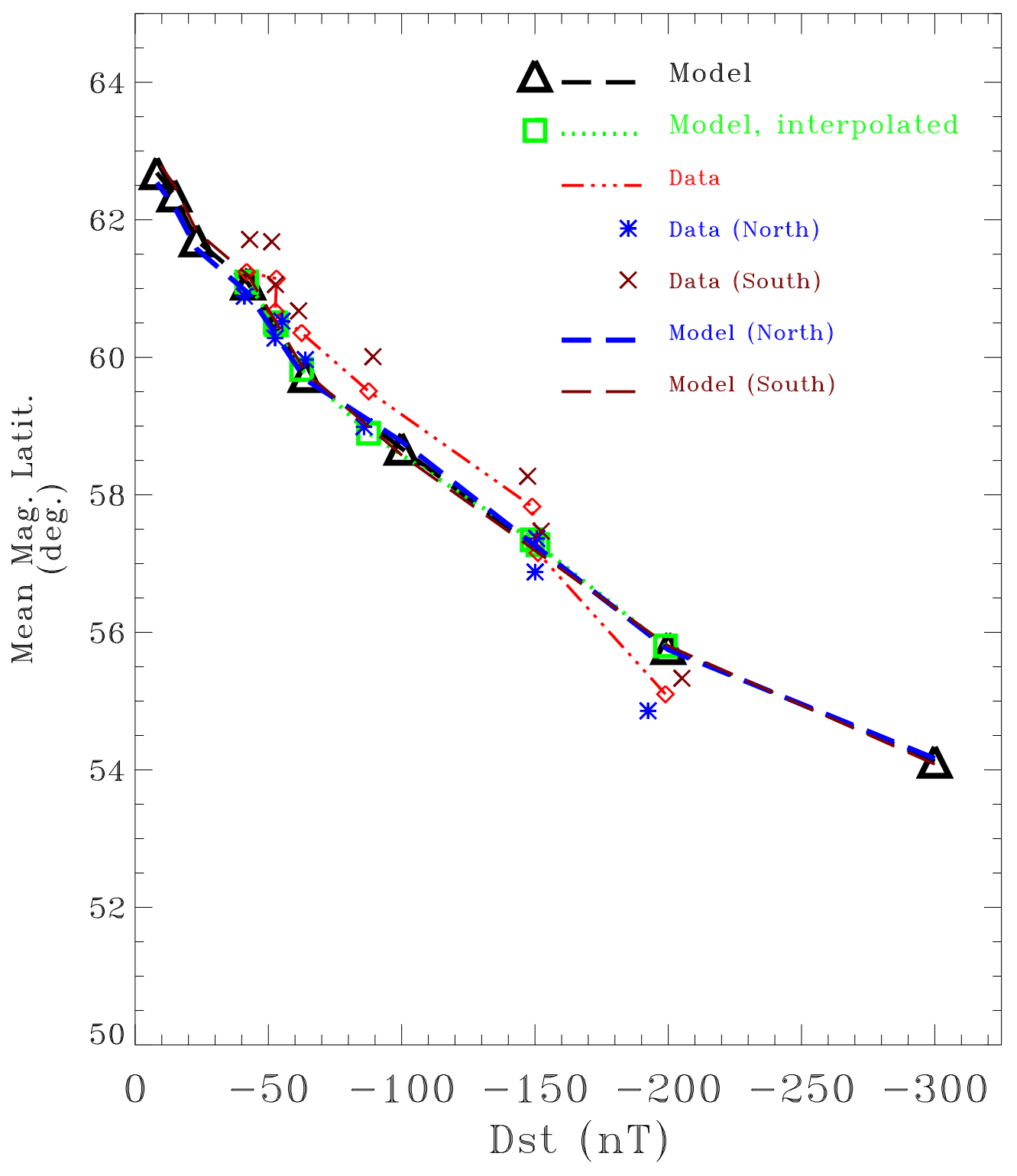


Figure 16: For the eight Kp bins of this work, comparisons of the mean measured magnetic latitudes at the mean measured Dst values (red diamonds) with the mean model magnetic latitudes at the model Dst values used for the Kp bins (black triangles). We also show interpolations of the mean model magnetic latitudes to the mean measured Dst values (green squares), see text for further description. The Northern and Southern hemisphere mean magnetic latitudes are also included. The Northern data are shown as blue asterisks, and the model results are shown as the blue curve. The equivalent Southern results are shown in brown. For clarity, we have omitted the symbols for the Northern and Southern hemisphere interpolated mean model magnetic latitude results. Note that all the model curves lie closely together, and for example, the black model curve is obscured by the overlying curves for much of the figure.

Print version: For the eight Kp bins of this work, comparisons of the mean measured magnetic latitudes at the mean measured Dst values (lighter diamonds and dashed - double dotted - dashed curve) with the mean model magnetic latitudes at the model Dst values used for the Kp bins (dark black triangles and thick, dark, dashed curve). We also show interpolations of the mean model magnetic latitudes to the mean measured Dst values (dark, thick squares and dotted curve), see text for further description. The Northern and Southern hemisphere mean magnetic latitudes are also included. The Northern data are shown as lighter asterisks, and the model results are shown as the lighter, thicker dashed curve. The equivalent Southern results are shown as lighter $\mathrm{X}$ symbols and lighter, thinner dashed curve. For clarity, we have omitted the symbols for the Northern and Southern hemisphere interpolated mean model magnetic latitude results. Note that all the model curves lie closely together, and for example, the thick, dark, dashed model curve is obscured by the overlying curves for much of the figure. 

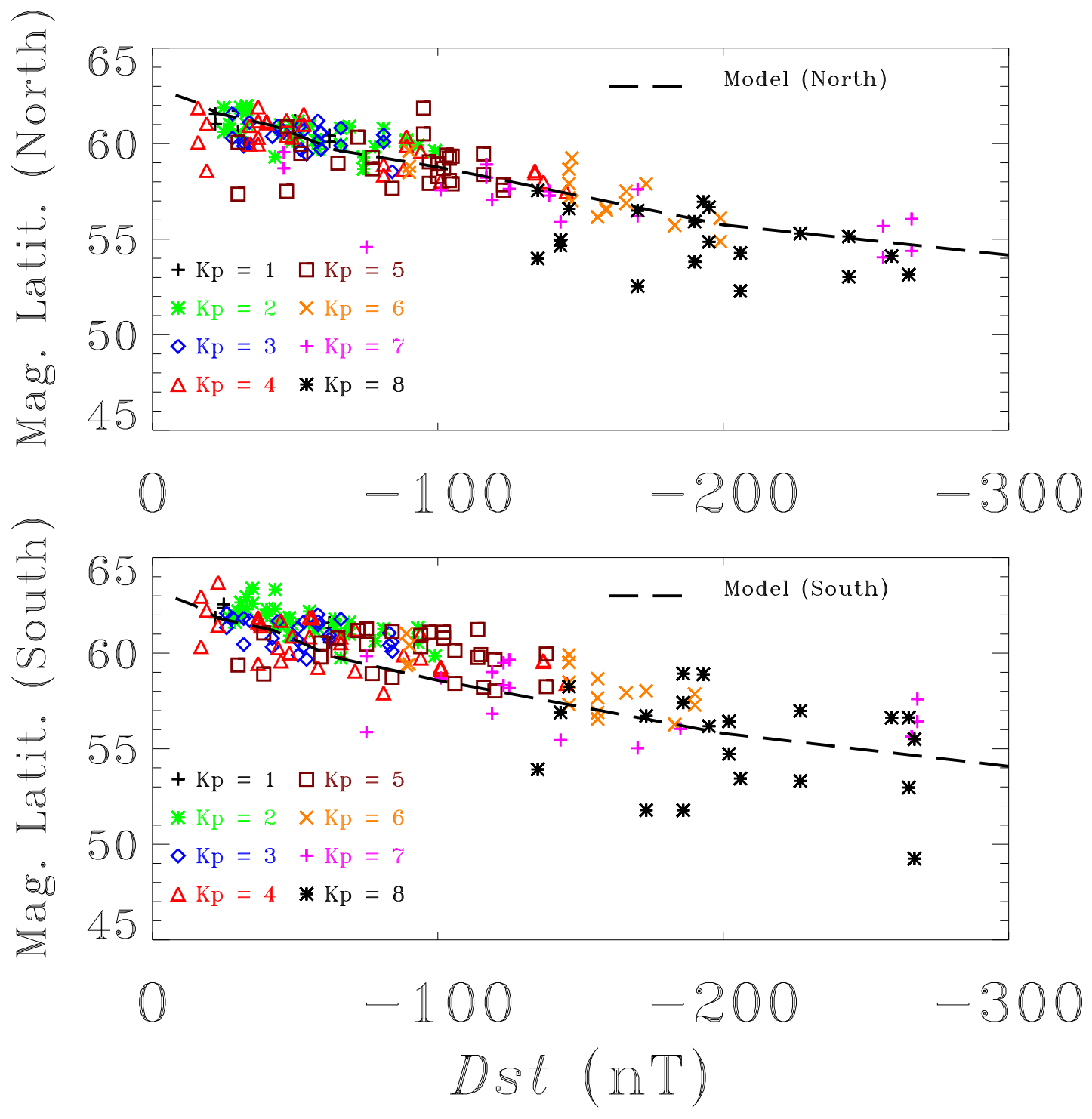
Figure 17: For the eight Kp bins, the observed magnetic latitudes are plotted against the observed Dst values for all the individual results of this work. The Northern hemisphere results are shown in the upper panel, and the Southern hemisphere results are shown in the lower panel. The measurements are coded by color/symbol pairs for each Kp level. For comparison, we also include the mean magnetic latitude results (black curves, model points not shown as separate symbols), joining the mean model calculations at the model Dst values (equivalent to the blue and brown curves in Figure 16 for the Northern and Southern hemispheres, respectively).

Print version: For the eight Kp bins, the observed magnetic latitudes are plotted against the observed Dst values for all the individual results of this work. The Northern hemisphere results are shown in the upper panel, and the Southern hemisphere results are shown in the lower panel. The measurements are coded by shading/symbol pairs for each Kp level. For comparison, we also include the mean magnetic latitude results (black curves, model points not shown as separate symbols), joining the mean model calculations at the model Dst values (equivalent to the thicker, lighter and thinner, lighter curves in Figure 16 for the Northern and Southern hemispheres, respectively). 